\title{
CHIRALITY AND SYMMETRY BREAKING IN A DISCRETE INTERNAL SPACE
}

\author{
Bodo Lampe
}

\begin{abstract}
In previous papers the permutation group $S_{4}$ has been suggested as an ordering scheme for elementary particles, and the appearance of this finite symmetry group was taken as indication for the existence of a discrete inner symmetry space underlying elementary particle interactions. Here it is pointed out that a more suitable choice than the tetrahedral group $S_{4}$ is the pyritohedral group $A_{4} \times Z_{2}$ because its vibrational spectrum exhibits exactly the mass multiplet structure of the 3 fermion generations. Furthermore it is noted that the same structure can also be obtained from a primordial symmetry breaking $S_{4} \rightarrow A_{4}$. Since $A_{4}$ is a chiral group, while $S_{4}$ is achiral, an argument can be given why the chirality of the inner pyritohedral symmetry leads to parity violation of the weak interactions.
\end{abstract}




\section{Introduction}

In the left-right symmetric standard model with gauge group $U(1)_{B-L} \times S U(3)_{c} \times$ $S U(2)_{L} \times S U(2)_{R}$ [1] there are 24 left-handed and 24 right-handed quark and lepton fields which including antiparticles amounts to 96 fermionic degrees of freedom, i.e. this model has right handed neutrinos as well as righthanded weak interactions.

In recent papers [2, 3, 4] a new ordering scheme for the observed spectrum of quarks and leptons was presented, which relies on the structure of the group of permutations $S_{4}$ of four objects, and a mechanism was proposed, how 'germs' of the Standard Model interactions emerge from this symmetry.

In those papers a constituent picture was suggested where quarks and leptons are assumed to be built from 'tetronic' constituents, whose interchanges generate the permutation group $S_{4}$. In the present paper I follow a different approach which relies on the fact that $S_{4}$ is not only the symmetry group of a regular tetrahedron, but can appear as the point group of several cubic lattices [5, 7]. In this approach the inner symmetry space is not continuous (with a continuous symmetry group) but has instead the discrete structure of a 3-dimensional cubic lattice 1 and the observed quarks and leptons can be interpreted as excitations on this lattice and characterized by representations of the lattice point group $S_{4}$.

In the following sections I will discuss in detail the nature of these excitations and relate them to the Standard Model phenomenology. I will further argue that the tetron model is is not just a strange observation in the sideways of particle physics, but has a more fundamental meaning and may shed light on some important issues of high energy physics. Actually, in modern particle physics there are several outstanding problems which have not been fully understood for many decades: they are the parity violation in weak interactions, the family structure of quarks and leptons, the calculation of their masses and CKM matrix elements and the existence of UV-divergences at very small distances.

\footnotetext{
${ }^{1}$ It seems then natural to assume that not only the internal symmetry is discrete but that physical space is a lattice, too. Although theories with a discrete inner symmetry over a continuous base manifold have been examined [8] they seem to me a bit artificial because they usually lead to domain walls and other discontinuities. Nevertheless, this point may be left open here, because for most arguments in this article it is not essential, whether physical space is discrete or continous.
} 
In my article I want to analyze these phenomena in the light of the tetron model. Tetron interactions will be assumed to describe a deeper level of matter than the Standard Model, somewhere between the Planck scale and the electroweak breaking scale.

The outline of the paper is as follows: section 2 describes the role of finite groups in tetron theory and shows how the correct multiplet structure of quarks and leptons can be obtained. In section 3 I elaborate on vibrational modes in the inner symmetry crystal (which will be named 'phinons'). I will show how the family structure of the Standard model fermions naturally arises in this framework. In addition I discuss the question whether the crystal can be compactified to an (inner) 'molecule'. Section 4 describes the Standard Model gauge symmetry as a phenomenon which arises from phinon-phinon interactions. Section 5 deals with a phase transition from an achiral to a chiral internal lattice. It will be shown that the corresponding symmetry breaking underlies the Standard Model Higgs mechanism. In section 6 the 7-dimensional cross product is introduced as a possible effective interaction between phinons, and it is shown that the chirality of the inner symmetry lattice induces the parity violation as found in the weak interactions. Finally, in section 7 an alternative scheme is presented which relies on spin waves instead of vibrational modes in the inner symmetry crystal.

\section{The Role of Finite Groups in Ordering the Spec- trum of Quarks and Leptons}

While $S_{4}$ has been discussed extensively in earlier papers, in this letter the focus will be on the so-called symmetric (or alternating) group $A_{4}$ which is the subgroup of $S_{4}$ consisting of even permutations of 4 objects. It is isomorphic to the group of proper rotations of a regular tetrahedron, and is therefore a 'chiral' symmetry (in the sense that it does not contain any inversions or reflections).

I will start with a simple pedagogical example: consider a tetrahedral molecule with 4 identical 'atoms' (one on each corner of the tetrahedron). In the first rows of table 1 the characters of $A_{4}$ are shown for the various classes and irreducible 


\begin{tabular}{|l|c|c|c|c|c|}
\hline & $\mathrm{I}$ & $4 C_{3}$ & $4 C_{3}^{2}$ & $3 C_{2}$ & $\begin{array}{c}\text { transformation behavior } \\
\text { of translations, rotations }\end{array}$ \\
\hline $\mathrm{A}$ & 1 & 1 & 1 & 1 & \\
$\mathrm{~A}$, & 1 & $e^{+2 i \pi / 3}$ & $e^{-2 i \pi / 3}$ & 1 & \\
$\mathrm{~A} "$ & 1 & $e^{-2 i \pi / 3}$ & $e^{+2 i \pi / 3}$ & 1 & \\
$\mathrm{~T}$ & 3 & 0 & 0 & -1 & $(x, y, z),\left(R_{x}, R_{y}, R_{z}\right)$ \\
\hline no. of atoms unchanged & 4 & 1 & 1 & 0 & \\
$\times \mathrm{T}$ & 12 & 0 & 0 & 0 & \\
$\mathrm{~A}+\mathrm{A}+\mathrm{A} "+3 \mathrm{~T}$ & 12 & 0 & 0 & 0 & \\
\hline
\end{tabular}

Table 1: Character table of the symmetric group $A_{4}$. I, $4 C_{3}, 4 C_{3}^{2}$ and $3 C_{2}$ are the classes consisting of the identity, 4 rotations by $2 \pi / 3$ and 4 rotations by $4 \pi / 3$ (each seen from a vertex) and 3 rotations by $\pi$. T is the vector representation, which acts on a regular tetrahedron in 3 dimensions, and A, A', A" can be traced back to representations of the cyclic group $Z_{3}$ (a subgroup of $A_{4}$ ).

representations [9]. In the fifth row for each class the number of atoms is given, which are left invariant by the corresponding transformations, and these numbers are multiplied in the sixth row by the characters of the translational representation $\mathrm{T}$ (which in the case of $A_{4}$ agrees with that for rotations, c.f. the last column of table 1). From the last row of the table one can conclude that the representation obtained in this procedure can be written as the sum $A+A^{\prime}+A^{\prime \prime}+3 T$ of irreducible representations.

Why am I doing this? The point is, that it is exactly the way phonon modes are classified for a lattice with $A_{4}$ symmetry, in which there are 4 'atoms' in the unit cell. The only difference with solid state physics being that I will be talking about a lattice and its vibrations which exist in a 3-dimensional internal symmetry space. For that reason the corresponding excitations will be called phinons.

If one passes from molecules to crystal structures there is a complication about which space group should be chosen. For the point group $A_{4}$, for example, there exist 6 space groups and their corresponding lattices, namely 195-199 corresponding to international standard symbols P23, F23, I23, P213, I213[5]. 
As a next step lattices with 8 atoms in the unit cell are considered. The procedure is similar as before. If, for example, the point group of the lattice is the full tetrahedral group $S_{4}$, there are 6 possible space groups (215-220 with international standard symbols P43m, F43m, I43m, P43n, F43c, I43d) [5]. Only for the choices 218, 219 and 220 one obtains the spectrum of phinon modes of the form

$$
A_{1}+A_{2}+2 E+3 T_{1}+3 T_{2}
$$

which was advocated in earlier papers [2, 3, 4]. In those papers the family structure of quarks and leptons was discussed on the basis of $S_{4}$ and expression (11).

In the case of space group 218 $(=\mathrm{P} 43 \mathrm{n})$ with 8 atoms in the unit cell one has Wyckoff positions 8e:

$$
\begin{aligned}
& W_{1}=(x, x, x) \quad W_{2}=(-x,-x, x) \quad W_{3}=(-x, x,-x) \quad W_{4}(x,-x,-x) \\
& W_{5}=(x+y, x+y, x+y) \quad W_{6}=(-x+y,-x+y, x+y) \\
& W_{7}=(-x+y, x+y,-x+y) \quad W_{8}=(x+y,-x+y,-x+y)
\end{aligned}
$$

where $y=\frac{1}{2}$. These points can be interpreted as 2 tetrahedrons shifted away from each other by the vector $(y, y, y)$.

For 219 and 220 one should use the Wyckoff positions 32e and 16c respectively, and take into account that for body centered $(\mathrm{BCC}=\mathrm{I})$ and face centered $(\mathrm{FCC}=\mathrm{F})$ space groups like 220 and 219 one usually does not use primitive unit cells but centered cells where the number of atoms is multiplied by the number of centering vectors (2 for I and 4 for F). For 219 (F43c) the resulting positions are the same as eq. (2) while for 220 (I43d) they are somewhat more complicated:

$$
\begin{aligned}
U_{1}=(x, x, x) & U_{2}=\left(-x+\frac{1}{2},-x, x+\frac{1}{2}\right) \\
U_{3}=\left(-x, x+\frac{1}{2},-x+\frac{1}{2}\right) & U_{4}\left(x+\frac{1}{2},-x+\frac{1}{2},-x\right) \\
U_{5}=\left(x+\frac{1}{4}, x+\frac{1}{4}, x+\frac{1}{4}\right) & U_{6}=\left(-x+\frac{1}{4},-x+\frac{3}{4}, x+\frac{3}{4}\right) \\
U_{7}=\left(x+\frac{3}{4},-x+\frac{1}{4},-x+\frac{3}{4}\right) & U_{8}=\left(-x+\frac{3}{4}, x+\frac{3}{4},-x+\frac{1}{4}\right)
\end{aligned}
$$

A disadvantage of $S_{4}$ is that it is an achiral symmetry (in the sense that it contains reflections). This will become an issue in section 6 where weak parity violation will 
be associated with a chiral inner symmetry. Furthermore, the lepton mass spectrum does not exactly fit into the representations (11). Muon and $\tau$-lepton as well as $\nu_{\mu}$ and $\nu_{\tau}$ would be in the same multiplet und retain equal masses.

Therefore an alternative based on the so-called pyritohedral symmetry $A_{4} \times Z_{2}$ will now be examined. Going from $A_{4}$ (table 1) to $A_{4} \times Z_{2}$ is a simple task because it amounts to replacing each of the representations $\mathrm{R}$ of table 1 by a combination of $R_{g}+R_{u}$ of odd and even vibrations under $Z_{2}$ and yields a multiplet spectrum of 24 vibrational modes:

$$
\begin{array}{r}
A_{g}(e)+A_{g}^{\prime}(\mu)+A_{g}^{\prime \prime}(\tau)+A_{u}\left(\nu_{e}\right)+A_{u}^{\prime}\left(\nu_{\mu}\right)+A_{u}^{\prime \prime}\left(\nu_{\tau}\right) \\
+T_{g}(u)+T_{g}^{\prime}(c)+T_{g}^{\prime \prime}(t)+T_{u}(d)+T_{u}^{\prime}(s)+T_{u}^{\prime \prime}(b)
\end{array}
$$

I have added in brackets the associated fermion flavor because the above combination of phinon modes for symmetry $A_{4} \times Z_{2}$ and 8 atoms in the unit cell will be interpreted as the set of 24 quark and lepton states. Note that the $Z_{2}$ factor $(u \leftrightarrow g$ in expression (44) ) corresponds to broken weak isospin and puts the weak isospin partners naturally in different multiplets with different masses.

In general, a crystal with $\mathrm{N}$ atoms is composed of $\mathrm{N} / \mathrm{n}$ primitive unit cells, each of which containing $\mathrm{n}$ atoms. For a d-dimensional crystal the total number of vibrational degrees of freedom is $d \times N$ which are distributed in $d \times n$ 'modes' or 'branches' throughout the first Brillouin zone. In the present case $(d=3, n=8)$ this amounts to the $3 \times 8=24$ modes of eq. (44). As a consequence there is the following simple memo: the number of families corresponds to the number of dimensions of the discrete inner symmetry space. The number of quarks and leptons within a family corresponds to the number of atoms in a unit cell of the inner symmetry lattice.

I have gone through the list of all finite point groups and found that there is no other group which is able to describe the mass multiplets as accurately as $A_{4} \times Z_{2}$. For example, the existence of a 3 dimensional representation is an essential requirement, if one wants to obtain equal masses for quarks with the same color, i.e. $m\left(q_{1}\right)=$ $m\left(q_{2}\right)=m\left(q_{3}\right)$, but among the 15 groups of order $24[10]$ only $A_{4} \times Z_{2}$ and $S_{4}$ have 3 dimensional representations 2

\footnotetext{
${ }^{2}$ The nontrivial groups of order 24 are $D_{8} \times Z_{3}$ and $\operatorname{Dic}_{1} 2 \times Z_{2}, S_{3} \times K, \operatorname{Dic}_{2} 4, \mathrm{SL}(2,3), Q_{8} \times Z_{3}$,
} 
There are in fact 7 crystallographic space groups with point group $A_{4} \times Z_{2}$ : 200206, corresponding to the international standard symbols Pm3, Pn3, Fm3, Fd3, Im3, Pa3, Ia3. All of them can have 8 atoms in the unit cell and lead to the required spectrum expression (44). Furthermore, as shown in section 5, all of them can be derived from breaking some larger space group with the octahedral group $O_{h}$ as its point group. For convenience at this point I just give the Wyckoff positions 8c for space group 205 (Pa3) with 8 atoms in the unit cell[5]:

$$
\begin{aligned}
C_{1}=(x, x, x) & C_{2}=(-x+y,-x, x+y) \\
C_{3}=(-x, x+y,-x+y) & C_{4}=(x+y,-x+y,-x) \\
C_{5}=(-x,-x,-x) & C_{6}=(x+y, x,-x+y) \\
C_{7}=(x,-x+y, x+y) & C_{8}=(-x+y, x+y, x)
\end{aligned}
$$

again with $y=\frac{1}{2}$.

One can get insight in the geometry of this configuration (which notably is realized in $\alpha N_{2}$ nitrogen) by considering the limiting cases $|x| \gg|y|$ and $|x| \ll|y|$. For very large $\mathrm{x}$ the system reduces to a cube with full (achiral) $O_{h}$ symmetry whereas for very small x one obtains 2 tetrahedrons lying above each other with full (achiral) $S_{4}$ symmetry. In section 5 symmetry breaking phase transitions will be considered which involve distortions of the internal lattice from these two limits to finite values of $\mathrm{x}$, and it will be shown that a symmetry breaking of the form $S_{4} \rightarrow A_{4}$ or $O_{h} \rightarrow A_{4} \times Z_{2}$ may have occured when the universe cooled down after the big bang. This symmetry breaking turns out to be the basis of the breaking of weak $\mathrm{SU}(2)$ in the Standard Model.

\section{$3 \quad$ Inner Lattice or inner Molecule?}

In the last section we have seen that the procedure for ordering the quark lepton spectrum is analogous to that for phonons in certain crystals, the only difference being that the crystal should lie in a 3-dimensional inner symmetry space and the displacements should go into those inner directions. For that reason these excitations have been named 'phinons'.

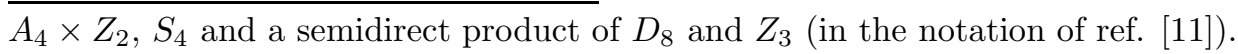


There may be other interpretations of the tetrahedral ordering like that the spectrum (4) can be obtained by using e.g. rotational instead of translational degrees of freedom, c.f the last column of table 1. In that case one is led to consider spin models [12, 13], and the vector displacements of the atomic vibrations are replaced by axial vectors $\vec{S}=\frac{1}{2} \psi^{\dagger} \vec{\tau} \psi$ which fulfil (internal) angular momentum commutation relations and can be considered to be built from an (internal) spinor field $\psi$. Phinons are replaced by 'mignons' in that context, quasi-particle excitations of the 3-dimensional inner spin vector $\vec{S}$ which obey the dynamics of a Heisenberg Hamiltonian. This alternative will be discussed later in sect. 7. At the present stage I want to stick to 'phinons' as the simplest and most effective solution, which will be seen to lead to the correct phenomenology.

The theory for phinons can be developed in analogy with the known results for phonons in solid state lattices. If one likes one may assume a situation where originally there was a discrete $(6+1)$-dimensional spacetime which by some compactification process splitted into an 3-dimensional internal space and a remaining $(3+1)$-dimensional physical spacetime so that the notions of time and energy and even temperature are defined for the inner symmetry lattice as well.

Consider then an inner crystal for any fixed base point on Minkowski space and allow for vibrations given by displacements of its atoms from their equilibrium position which are assumed to be orthogonal to the base space. One can then expand the potential energy function around the minimum energy configuration. The leading term is the ground state energy $E_{0}$ and the next-to-leading term is quadratic in the displacements $\vec{u}(l, s)$ of the atoms $\mathrm{s}$ in a unit cell $\mathrm{l}$ :

$$
U=E_{0}+\frac{1}{2} \sum_{l, s, i, l^{\prime}, s^{\prime}, j} k_{i j}\left(l, s, l^{\prime}, s^{\prime}\right) u_{i}(l, s) u_{j}\left(l^{\prime}, s^{\prime}\right)
$$

where $i, j=1,2,3$ are vector indices of the internal space and $k_{i j}\left(l, s, l^{\prime}, s^{\prime}\right)$ is the so called force or spring constant matrix[14] which by definition relates the forces to the displacements. Note that s,s' run from 1 to $n$ and l,l' from 1 to $\mathrm{N} / \mathrm{n}$, where $\mathrm{N}$ is the total number of atoms in the crystal.

For the calculation of the phonon spectrum there are two strategies. One is to use the available computer routines [14, 15], and the other to transform to normal coordinates and then try approximations. For example, from the geometrical description given 
after eq. (5) one may deduce an ansatz which simplifies the equations very much, namely one is lead to assume that there are essentially only two different spring constants: one $\left(k_{1}\right)$ connecting two atoms within a tetrahedron, and the other one $\left(k_{2}\right)$ connecting the 2 atoms within a $Z_{2}$ pair. $k_{2}$ may then also be considered the coupling strength between two tetrahedrons. In the next step one can try a strong coupling expansion where one assumes that one of the couplings is much smaller than the other and expands the phinon frequencies in powers of $k_{1} / k_{2}$ or $k_{2} / k_{1}$, respectively. Such a calculation can be performed, for example, in the mean field approximation.

Due to the translational invariance of the crystal, the vibrational modes must be characterized by an additional parameter: their wave-vector $\vec{q}$. It is defined by the relation

$$
u_{i}(l, s)=u_{i}(\vec{q}, s) e^{i[\vec{q} r(l, s)-\omega t]}
$$

where $i=1,2,3$ and $\vec{r}(l, s)$ represents the equilibrium position of atom $\mathrm{s}$ in primitive cell 1. Using this as an ansatz one may solve the equations of motion and gets $3 \mathrm{n}$ solutions with frequencies $\omega(\vec{q}, \alpha), \alpha=1, \ldots, 3 n$, where $\mathrm{n}$ is the number of atoms in the unit cell. For an infinite crystal a band structure is obtained for each mode.

The total vibrational free energy $V_{F}=\sum_{\alpha} V_{\alpha}$ is a sum of contributions from the modes $\alpha$ which are given by

$$
V_{\alpha}=k_{B} T \sum_{\vec{q}} \ln \left(2 \sinh \frac{\hbar \omega(\vec{q}, \alpha)}{2 k_{B} T}\right)
$$

where $\omega(\vec{q}, \alpha)$ is the frequency of the $\alpha$-th mode at wave-vector $\vec{q}$. In an infinite crystal one should instead consider the free energy per primitive cell, also called $V_{\alpha}$ here and given by:

$$
V_{\alpha}=\frac{k_{B} T V}{(2 \pi)^{3}} \int_{B Z} d^{3} q \ln \left(2 \sinh \frac{\hbar \omega(\vec{q}, \alpha)}{2 k_{B} T}\right)
$$

At low temperatures these expressions, which are non-analytic in $\mathrm{T}$, have only a very weak temperature dependence. In fact at small T eq. (8) can be approximated by

$$
V_{\alpha}(T \rightarrow 0)=\frac{1}{2} \sum_{\vec{q}} \hbar \omega(\vec{q}, \alpha)+k_{B} T \exp \left[-\frac{\hbar \omega(\vec{q}, \alpha)}{k_{B} T}\right]
$$


Note that since I want to identify quarks and leptons with the vibrational modes, eq. (10) is a direct measure of their mass, at least in this idealized situation of a translationally invariant internal crystal. One may speculate at this point about the definition of temperature in the inner symmetry space and whether the same Boltzmann constant should be used there to transform 'Kelvin' units into energy. A related question is how and if at all entropy is exchanged between internal and physical space. In any case, the momenta of phinons excited at present days energies are rather small as compared to the extension of the internal Brillouin zone, with the dependence on temperature being very weak. We are therefore mostly concerned with the special case of $\vec{q}=0$, the vibrational behavior of the crystal at the center of the Brillouin zone, the so-called $\Gamma$-point. For the nonlinear process of scattering of two phinons discussed in section 4 one should in principle consider balanced composite states where one phinon has momentum $+\vec{q}$ and the other one $-\vec{q}$ (cf. eq. (43) $)$, and this will involve representations of space groups at general points $\vec{q}$. A plane wave in our full $3+3+1$ dimensional model is a Bloch wave

$$
\Psi_{i a}=f_{a}(\vec{x}) u_{\alpha}(\vec{r}) e^{i(\vec{p} \vec{x}-\nu t)} e^{i(\vec{q} \vec{r}-\omega t)}
$$

where the amplitude $u$ has the periodicity of the internal lattices and the amplitude $\mathrm{f}$ has the periodicity of the lattice on physical space (provided such a lattice exists, c.f. footnote 1). It is a bosonic amplitude with respect to internal space (vector $\vec{r}$, index $\alpha$ and momentum $\vec{q}$ for modes $\alpha=1, \ldots, 24)$ and a fermion wave with respect to the lattice on physical space (vector $\vec{x}$, index a and momentum $\vec{p}$ ) whose spacing however is assumed to be too small to be observable experimentally, so that Poincare invariance may be safely assumed. If one wants to discuss effects from the lattice structure in physical space, one should choose the Bloch functions in such a way that they transform according to the spin- $\frac{1}{2}$ representation of the relevant point group of the physical lattice. This 2-dimensional representation is usually called $G_{1}$, and its effects within the framework of the tetron model have been analyzed in ref. [16].

The spinor index a runs from 1 to 2 or from 1 to 4 , depending on whether one considers relativistic effects or not. For simpler understanding I usually restrict myself to the nonrelativistic picture. However, by using Dirac fields instead of 2component Pauli spinors one may easily include antiparticles in the considerations. Considering plane waves for the internal vibrations may be dangerous in view of 
the fact that nothing is known about the forces acting between lattice points, and at later stages strong anharmonicity properties will indeed be assumed. However, Bloch waves do not have this problem because Blochs theorem presupposes only translational invariance and holds independently of the nature of the forces 3

Nevertheless, it is difficult to estimate the physical significance of the internal wave vector $\vec{q}$ and the associated dispersion. In eq. (9) $\vec{q}$ appears as an integration variable, i.e. it is integrated out when it comes to the calculation of total energies as measured from physical space. Geometrically, $\vec{q}$ corresponds to a translatory motion of the unit cell on the inner symmetry lattice, and one may therefore justly ask the question, how extended the inner lattice is, i.e. whether it is infinite (with a dependence of the modes on a continuous $\vec{q}$, which an observer on the base space cannot measure) or if it consists only of a few unit cells, e.g. with periodic boundary conditions. What I have in mind is the idea, that through some compactification process the crystal consists maybe of only one unit cell, in which case there is trivially only one wave vector in the first Brillouin zone and the 'crystal' resembles more an inner molecule. As discussed earlier, a 3-dimensional lattice with 8 atoms in the unit cell has 24 vibrational modes. However an 8-atomic molecule has only 18 of them, where the reduction by 6 is due to lacking translational and rotational degrees of freedom. A way out in order to keep the 24 modes is to assume that the single compact unit cell is physically fixed on its Minkowski base point with no large translations or rotations possible. In that case the 6 translations and rotations re-establish to become vibrational modes again.

The assumption of an internal molecule fixed to its base point has several advantages over a large internal crystal. Not only one can do without internal wave vectors, but one also avoids the problem that in a large internal crystal the phinons would dissipate to infinity, while in a smaller discrete structure they will remain localized and perpetual excitations. Furthermore, it is difficult to imagine how a large internal crystal is 'fixed' to a base point in a way that translational invariance (and the validity of Blochs theorem) is not destroyed, because internal atoms which are far away from the base point may feel much weaker forces than those near to the base.

\footnotetext{
${ }^{3}$ Note again that we are talking about Bloch waves in internal space while the Bloch waves in physical space reduce to ordinary free fermion fields in the limit of large distances (small spatial lattice constants).
} 
A possible way out is that one works with a finite number of unit cells which are distributed symmetrically with respect to their attractive base point so that one can use periodic boundary conditions. In that case one has only a finite number of q-values to consider, and the sums e.g. in eq. (8) go over those values.

Note that these considerations also affect the question of acoustic modes and massless excitations. Usually, in phonon theory the energy of 3 of the $3 \mathrm{n}$ phonon branches goes to zero as $\vec{q} \rightarrow 0$. These are called the acoustic modes and are equivalent to pure translations of the crystal. According to the last column of table 1 in the present case they correspond to one of the $T_{g}$ representations in eq. (44). However, if the finite internal crystal or the one-cell molecule is fixed to the base point by some harmonic force the acoustic modes will acquire a non-zero energy even at $\mathrm{q}=0$.

The question lattice or single unit cell will be left open for the rest of the paper. Although I find the one-unit-cell option somewhat simpler to imagine, because in that case each real point in physical space just splits into 8 vibrating atoms, I will often concentrate on the phinon picture with more than one unit cell, because it has a richer phenomenology and also allows for phase transitions.

\section{Gauge Symmetries from Phinon Scattering}

If one accepts the above ideas about fermions, the immediate question is how the gauge interactions can arise from a discrete inner symmetry.

In the present context they are to be interpreted as anharmonic phinon scattering states, with the full gauge symmetries appearing as emergent phenomena. Just as the quarks and leptons themselves the gauge bosons are vibrational excitations which however arise from nonlinear forces between the atoms of the inner symmetry lattice, corresponding to anharmonic terms typically of fourth order in the phinon interactions which in turn allow for scattering processes among the phinons. Thus the emergence of gauge structures consists in two steps: firstly, the gauge bosons arise as bound states from the scattering processes of phinons, and secondly their effective interactions should follow a gauge symmetry, in order to keep track of the 'connection' and the differential geometry of the full $3+3+1$ dimensional 'fibre space'. 
A similar point of view has been taken in ref. 2] where it was shown that on a group theoretical level gauge bosons can be constructed as 'Cooper states' of the quark and lepton excitations. The idea is in fact not unusual and is used both in relativistic particle physics [17, 18, 19, 20] and in non-relativistic statistical models [22, 21, 23, 26] with continuous as well as with discrete base spaces. In the present framework the starting point would be the nonlinear phinon and mignon interactions eqs. (41) and (83) given below which lead to scattering processes in the internal crystal. On the base space this will induce an effective Lagrangian of the generic form

$$
L=\sum_{n, m} \bar{f}(\vec{n}) \gamma^{\mu} G_{\mu}^{a}(\vec{n}, \vec{m}) \lambda^{a} f(\vec{m})
$$

where $\gamma_{\mu}$ are the ordinary Dirac matrices in $3+1$ dimensions, $\lambda^{a}$ the generators of the local gauge group and the sum is over (base space) lattice sites $\mathrm{n}$ and $\mathrm{m}$. The interacting field is constructed as

$$
G_{\mu}^{a}(\vec{n})=<0\left|\bar{f}(\vec{n}) \gamma_{\mu} \lambda^{a} f(\vec{m}+\vec{\mu})\right| 0>
$$

(with $\vec{\mu}$ being the next lattice point in the $\mu$-direction) and behaves as

$$
G_{\mu}(\vec{n}) \rightarrow U(\vec{n}) G_{\mu}(\vec{n}) U^{\dagger}(\vec{m}+\vec{\mu})
$$

under local gauge transformations while fermions transform as

$$
f(\vec{n}) \rightarrow U(\vec{n}) f(\vec{n})
$$

It can then easily be proven that the Lagrangian eq. (12) is gauge invariant. The method has also been used [23, 21, 22, to prove the equivalence of Heisenberg spin models with certain gauge theories and can indeed be generalized: if the scattering states exist, then gauge groups with a continuous symmetry appear as necessary by-products of the model. For the case of the tetron model these arguments have been presented in detail in ref. [2], and I do not want to repeat them here for the complete Standard Model, but just sketch the prove for the simpler case of QCD interactions: from eq. (4) one sees that quarks always appear as triplets. One would like to identify these triplets with $\mathrm{SU}(3)$ color triplets. In order that to be possible one has to assume that gauge symmetries and interactions are emergent 
effects which arise from the originally discrete symmetry. In a first step one may analyze the symmetry content of a $2 \rightarrow 2$ scattering of $A_{4}$-triplets:

$$
T \otimes T=A+A^{\prime}+A^{\prime \prime}+2 T
$$

Using the symmetry adapted functions for the representations $\mathrm{T}$ on the left hand side of (16) and evaluating the corresponding Clebsch-Gordon coefficients leads to

- a representation of the $(B-L)$-photon as

$$
B_{\mu}=\bar{u}_{1} \gamma_{\mu} u_{1}+\bar{u}_{2} \gamma_{\mu} u_{2}+\bar{u}_{3} \gamma_{\mu} u_{3}
$$

which originates from the representation $A$ on the right hand side of eq. (16) and from the corresponding Clebsch-Gordon coefficient [9]

$$
V(T, T, A ; i, j, 1)=\frac{1}{\sqrt{3}} \delta_{i j}
$$

- a representation of the gluon octet stemming from the remaining part $A^{\prime}+$ $A^{\prime \prime}+2 T$ of the decomposition eq. (16). Namely, the CG-coefficients can be written in terms of the Gell-Man $\lambda$-matrices as

$$
\begin{aligned}
V\left(T, T, A^{\prime} ; i, j, 1\right) & =\frac{1}{2} \lambda_{8 i j} \\
V\left(T, T, A^{\prime \prime} ; i, j, 1\right) & =\frac{1}{2} \lambda_{3 i j} \\
V(T, T, T ; i, j, k) & =\frac{1}{\sqrt{6}} \epsilon_{i j k} \\
& =\frac{i}{\sqrt{6}} \lambda_{7,5,2 i j} \quad \text { for } \quad k=1,2,3
\end{aligned}
$$

where the last expression corresponds to the first copy of $\mathrm{T}$ on the right hand side of eq. (16) and

$$
\begin{aligned}
V(T, T, T ; i, j, k) & =\frac{1}{\sqrt{6}}\left|\epsilon_{i j k}\right| \\
& =\frac{1}{\sqrt{6}} \lambda_{6,4,1 i j} \quad \text { for } \quad k=1,2,3
\end{aligned}
$$

to the second. All in all we obtain

$$
\begin{aligned}
G_{3 \mu} & =\bar{q}_{1} \gamma_{\mu} q_{1}-\bar{q}_{2} \gamma_{\mu} q_{2} \\
G_{8 \mu} & =\frac{1}{\sqrt{3}}\left(\bar{q}_{1} \gamma_{\mu} q_{1}+\bar{q}_{2} \gamma_{\mu} q_{2}-2 \bar{q}_{3} \gamma_{\mu} q_{3}\right)
\end{aligned}
$$


and similarly for the other $\lambda$-matrices.

The fact that formally the same bilinear combinations are created as needed in $S U(3)_{\text {color }}$ QCD is no accident but has to do with the fact that $S_{4}$ and $A_{4}$ may be considered as subgroups of $\mathrm{SO}(3) \subset \mathrm{SU}(3)$. The result is therefore an elaboration on the claim formulated in [3] that the appearant tetrahedral symmetry of quarks and leptons is able to provide 'germs' of the Standard Model interactions, which are necessarily gauge interactions because these are the only consistent quantum field theories fulfilling the appropriate symmetry requirements (at energies small as compared to the inverse lattice spacings).

Similarly the weak bosons can be obtained from the $Z_{2}$ factor of the pyritohedral group as $g \otimes g, g \otimes u-u \otimes g$ and $u \otimes u$, with an additional contribution $g \otimes u+u \otimes g$ to the $(B-L)$-photon. A tricky point, however, is to understand parity violation of the weak interactions, i.e. the fact that only lefthanded fermions take part in the corresponding processes. This will be treated in section 6 , where a relation between the chirality of the internal lattice and weak parity violation will be constructed.

Another complication is the possible presence of an internal wave vector. If the internal crystal consists only of one unit cell, the above analysis is complete and there is nothing more to be said. However, if it is an extended crystal with wave vectors $\mathrm{q}$, the phinon-phinon interactions, which lead to the gauge fields, will involve scattering processes $(\vec{q}) \otimes(-\vec{q})$. This will be made concrete in the next section where the nonlinear terms responsible for the scattering will be written down explicitly (cf. eq. (41)). As a consequence, the group theoretical analysis presented above will become more complicated, because one has to consider representations of the full space group and not just of the point group $A_{4} \times Z_{2}$. These depend on the value of $\vec{q}$, on its star and the associated little groups, and the corresponding tensor products must be looked up, for example, on the Bilbao crystallographic server $[5,6]$. 


\section{Chirality and the Breakdown of the Tetrahedral Symmetry}

In the Standard Model the quark and lepton masses together with the KobayashiMaskawa mixing elements are free parameters, while they should be calculable in the tetron model as vibrational energies of phinon modes.

Experimentally there is a large difference in the masses of fermions, starting from (almost) massless neutrinos to the top quark mass. There are large differences between the families, but also within one family, and most noticeable even among weak isospin partners. The corresponding breaking of weak $\mathrm{SU}(2)$ does not mean that pyritohedral $A_{4} \times Z_{2}$ is a broken symmetry, too. On the contrary, it is unbroken, because it was constructed in such a way that all different mass particles can be ordered in different mass multiplets of this point group (cf. eq. (44)).

A natural question to ask is how a rather ugly symmetry like $A_{4} \times Z_{2}$ may have arisen to become the fundamental ordering principle of nature. After all, $A_{4} \times Z_{2}$ (and also $A_{4}$ ) is a chiral point group, and a crystal with such a symmetry always shows a chirality structure in the form of a (left or right handed) helical arrangement of the crystallic atoms. Therefore it seems reasonable to assume that $A_{4} \times Z_{2}$ itself arose from a primordial symmetry breaking within the inner symmetry crystal, i.e. that soon after the big bang there was a second order phase transition from a high-temperature phase with a larger lattice symmetry (tetrahedral $S_{4}$ or even the complete octahedral group $O_{h}$ ) to the present $A_{4} \times Z_{2}$ or $A_{4}$ phase. Correspondingly, the original multiplets would break up as

$$
\begin{gathered}
A_{2 g}+A_{2 u}+E_{u}+E_{g}+2 T_{1 g}+2 T_{1 u}+T_{2 g}+T_{2 u} \\
\rightarrow A_{g}+A_{g}^{\prime}+A_{g}^{\prime \prime}+3 T_{g}+A_{u}+A_{u}^{\prime}+A_{u}^{\prime \prime}+3 T_{u}
\end{gathered}
$$

for the breaking $O_{h} \rightarrow A_{4} \times Z_{2}$ leading to the desired fermion spectrum eq. (44) or according to

$$
A_{1}+A_{2}+2 E+3 T_{1}+3 T_{2} \rightarrow 2\left(A+A^{\prime}+A^{\prime \prime}+3 T\right)
$$

for $S_{4} \rightarrow A_{4} 4$ Note that the left hand side of eq. (28) corresponds to expression

\footnotetext{
${ }^{4}$ An alternative to spontaneous symmetry breaking is an explicit breaking of symmetries, which
} 
(1) while the left hand side of eq. (27) is obtained in a similay manner for a crystal with point group $O_{h}$ and 8 atoms in the unit cell.

How do these symmetry breakings come about? Since we have identified phinons as the relevant excitations it is tempting to suppose that we have to look for a displacive phase transition on the internal symmetry lattice, i.e. a spontaneous effect where the crystal is deformed by one phinon 'freezing' out at a critical temerature $T_{c}$ [27, 28, 29]. For this to happen, one needs a strongly anharmonic interaction, because the phinon frequency must fall to zero at the critical point, and then harden again below it, as the crystal finds a new equilibrium around the deformed structure.

Note that in the present case such a transition, which may or may not be accompanied by a compactification of the lattice (as discussed in the previous section), would be from a non-chiral to a chiral inner symmetry lattice. In the following this process will be described in some detail: consider the universe shortly after the big bang where the temperatures were high and the world was in a phase of high symmetry with all sorts of phinons excited. When the temperature fell, the frequency of one (or some) of the modes decreased, and when its value within the inner symmetry crystals fell below the critical point, this mode froze out to zero frequency and transformed to a static displacement pattern, thus inducing a transition to $A_{4} \times Z_{2}$ or $A_{4}$ and a shift in the Wyckoff positions to their present values.

Displacive phase transitions are well known in solid state physics and are usually described in terms of the normal coordinates $u$ used to describe the soft vibrational modes. In other words, the order parameter to be chosen is the displacement vector of the mode which freezes out at the phase transition, or, more generally, the mean displacement

$$
\eta=\frac{1}{N} \sum \vec{u}(l, s)
$$

could be induced for example by a pseudoscalar chiral interaction among the lattice atoms. Such an interaction is given e.g. by the scalar triple product

$$
H_{3}=f_{3} \sum_{l, l^{\prime}, l^{\prime \prime}, s, s^{\prime}, s^{\prime \prime}} \vec{u}(l, s)\left[\vec{u}\left(l^{\prime}, s^{\prime}\right) \times \vec{u}\left(l^{\prime \prime}, s^{\prime \prime}\right)\right]
$$

of lattice vectors which is positive for even permutations and negative for odd ones. In the philosophy discussed in the main text, however, eq. (29) is merely an effective interaction which could arise after the spontaneous symmetry breaking and may be used to describe chiral effects in the low energy regime. 
A simpler definition can be obtained by comparing the fractional coordinates of the atoms in the low-temperature and high-temperature phases. This has the further advantage that one circumvents all problems associated with thermal expansion which arise when using absolute displacements. For example, if atoms with fractional coordinates $\mathrm{x}, \ldots$ in the low-temperature phase tend towards $\frac{1}{4}, \ldots$ on heating to the high-temperature phase a natural order parameter would be

$$
\eta=\frac{1}{4}-x
$$

If, on the other hand, a transition involves rotations of a group of atoms, the order parameter can just as well be defined as the angle by which the group of atoms has rotated to break the symmetry. In leading order and for small displacements all these definitions will be equivalent. However, some of the displacements and rotations may follow the order parameter to higher order. Therefore it is better not to simply average over anything as in eq. (30), but to select those which are important in the formation of the new structure.

In the case at hand the freeze out proceeds in such a way that the achiral reflection symmetries gets lost:

- For $S_{4} \rightarrow A_{4}$ : the symmetric group $A_{4}$ is the group of proper rotations of a equilateral tetrahedron, while the tetrahedral group $S_{4}$ contains rotoreflections in addition. If $S_{4}$ is interpreted as the group of permutations of 4 objects, these rotoreflections correspond to the odd permutations in $S_{4}$, while $A_{4}$ contains even permutations only. The possible phase transitions for crystals with 8 atoms in the unit cell are: $218(\mathrm{P} 43 \mathrm{n}) \rightarrow 195(\mathrm{P} 23), 219$ (F43c) $\rightarrow 196(\mathrm{~F} 23)$ and 220 (I43d) $\rightarrow 199$ (I213) [6]. For example, in case 218 according to eq. (2) the symmetric high temperature phase is characterized by 2 tetrahedrons in the unit cell, both of extension $\mathrm{x}$, which are shifted uniformly by a vector $\left(\frac{1}{2}, \frac{1}{2}, \frac{1}{2}\right)$ whereas the broken phase is characterized by a transition of Wyckoff positions $8 e \rightarrow 4 e_{1}+4 e_{2}$ with $x_{1}=x$ and $x_{2}=x+\frac{1}{2}$, i.e. one is forced to introduce 2 types of Wycks in order to obtain the 2 required sets of representations $A+A^{\prime}+A^{\prime \prime}+3 T$. There is a similar situation for space groups 219 and 220 where one finds $32 e \rightarrow 16 e_{1}+16 e_{2}$ and $16 c \rightarrow 8 a_{1}+8 a_{2}$, respectively (cf. eq. (3)) . 
- For $O_{h} \rightarrow A_{4} \times Z_{2}\left(\right.$ or $\left.O_{h} \rightarrow A_{4}\right)$ : the octahedral group $O_{h}=O \times P_{i n}$ is in fact the direct product of proper rotations of a cube and the (inner) parity operation $P_{i n}$, so that the breaking to $A_{4} \times Z_{2}$ includes a breaking of $P_{i n}$. This breaking has the further advantage, that for a proper choice of space groups one can come along with only one type of Wyckoff positions. I have systematically scanned all space groups and Wyckoff positions in question and found only two solutions which fulfill all requirements:

- Firstly there is

$$
32 b(228=F d 3 c) \rightarrow A_{2 g} \rightarrow 32 e(203=F d 3)
$$

where the representation between the arrows denotes the mode that freezes out at the phase transition. For the starting configuration $228=\mathrm{Fd} 3 \mathrm{c}$ (32b) the Wyckoff positions are

$$
\begin{aligned}
F_{1} & =\left(\frac{1}{4}, \frac{1}{4}, \frac{1}{4}\right) & F_{2} & =\left(0, \frac{1}{2}, \frac{3}{4}\right) \\
F_{3} & =\left(\frac{1}{2}, \frac{3}{4}, 0\right) & F_{4} & =\left(\frac{3}{4}, 0, \frac{1}{2}\right) \\
F_{5} & =\left(\frac{3}{4}, \frac{3}{4}, \frac{3}{4}\right) & F_{6} & =\left(0, \frac{1}{2}, \frac{1}{4}\right) \\
F_{7} & =\left(\frac{1}{2}, \frac{1}{4}, 0\right) & F_{8} & =\left(\frac{1}{4}, 0, \frac{1}{2}\right)
\end{aligned}
$$

On cooling below the critical temperature these will go over to the positions

$$
\begin{aligned}
G_{1}=(x, x, x) & G_{2} & =\left(-x+\frac{1}{4},-x+\frac{1}{4}, x\right) \\
G_{3}=\left(-x+\frac{1}{4}, x,-x+\frac{1}{4}\right) & G_{4} & =\left(x,-x+\frac{1}{4},-x+\frac{1}{4}\right) \\
G_{5}=(-x,-x,-x) & G_{6} & =\left(x+\frac{3}{4}, x, x+\frac{3}{4},-x\right) \\
G_{7}=\left(x+\frac{3}{4},-x, x+\frac{3}{4}\right) & G_{8} & =\left(-x, x+\frac{3}{4}, x+\frac{3}{4}\right)
\end{aligned}
$$

of $\mathrm{Fd} 3=203(32 \mathrm{e})$ with point group $A_{4} \times Z_{2}$. The representations describing the phinon modes transform as given in eq. (27) and the order parameter may be chosen as anticipated in eq. (31).

- Secondly there is

$$
16 b(230=I a 3 d) \rightarrow A_{2 g} \rightarrow 16 c(206=I a 3)
$$


In this case the higher symmetry phase $230=\mathrm{Ia} 3 \mathrm{~d}(16 \mathrm{~b})$ has the configuration

$$
\begin{aligned}
D_{1} & =\left(\frac{1}{8}, \frac{1}{8}, \frac{1}{8}\right) & D_{2} & =\left(\frac{3}{8}, \frac{7}{8}, \frac{5}{8}\right) \\
D_{3} & =\left(\frac{7}{8}, \frac{5}{8}, \frac{3}{8}\right) & D_{4} & =\left(\frac{5}{8}, \frac{3}{8}, \frac{7}{8}\right) \\
D_{5} & =\left(\frac{1}{8}, \frac{1}{8}, \frac{1}{8}\right) & D_{6} & =\left(\frac{3}{8}, \frac{7}{8}, \frac{5}{8}\right) \\
D_{7} & =\left(\frac{7}{8}, \frac{5}{8}, \frac{3}{8}\right) & D_{8} & =\left(\frac{5}{8}, \frac{3}{8}, \frac{7}{8}\right)
\end{aligned}
$$

which on cooling below the critical temperature go over to the positions

$$
\begin{array}{rlrl}
E_{1}=(x, x, x) & E_{2} & =\left(-x+\frac{1}{2},-x, x+\frac{1}{2}\right) \\
E_{3}=\left(-x, x+\frac{1}{2},-x+\frac{1}{2}\right) & E_{4} & =\left(x+\frac{1}{2},-x+\frac{1}{2},-x\right) \\
E_{5}=(-x,-x,-x) & E_{6}=\left(x+\frac{1}{2}, x,-x+\frac{1}{2}\right) \\
E_{7}=\left(x,-x+\frac{1}{2}, x+\frac{1}{2}\right) & E_{8}=\left(-x+\frac{1}{2}, x+\frac{1}{2}, x\right)
\end{array}
$$

so that the order parameter may be chosen to be

$$
\eta=\frac{1}{8}-x
$$

The phase transitions described above will remove all degenericies between isospin partners, as contained in the representations E on the left hand sides of (27) and (28). Since the weak vector bosons are excitations between isospin partners and therefore between odd and even permutation states, the symmetry breaking will give masses to $\mathrm{W}$ and $\mathrm{Z}$ boson as well and thus will eventually be responsible for the $S U(2)_{L}$ breaking which in the Standard Model is described by the Higgs mechanism. Using the displacement of the freeze out mode as order parameter $\eta$ one may apply the Landau theory of phase transitions to obtain the symmetry breaking part of the free energy which in general reads

$$
V(\eta)=-\frac{1}{2} f_{2} \eta^{2}+\frac{1}{4} f_{4} \eta^{4}+O\left(\eta^{6}\right)
$$

with a positive parameter $f_{4}$ and $f_{2}=a\left(T-T_{c}\right)$ being linear in $T-T_{c}$ and positive for $\mathrm{T}$ larger than $T_{c}$. Odd powers of $\eta$ are excluded from eq. (39) by symmetry 
arguments and higher order terms can usually be neglected. The magnitude of the distortion in this potential is given by

$$
\left|\eta_{0}\right|=\sqrt{\frac{f_{2}}{f_{4}}}
$$

On the microscopic level the Landau free energy eq. (39) can be interpreted as the local double-well potential in which the inner atoms move and which is the driving force behind the phase transition. Originally it comes from anharmonic terms in the Lagrangian and is responsible for the freeze out of the soft mode. For a proper treatment the analysis should be extended to include all modes, i.e. all displacement coordinates. For simplicity I will assume that a transformation to normal coordinates has been performed and that these are given in momentum space as $u(\vec{q}, \alpha), \alpha=1, \ldots, 3 n$ with frequencies $\omega(\vec{q}, \alpha)$. If anharmonic interactions for all modes are included to fourth order, the interaction part of the Hamiltonian is given by

$$
\begin{aligned}
H_{W}= & \frac{1}{2} \sum_{\vec{q}, \alpha} \omega^{2}(\vec{q}, \alpha) u(\vec{q}, \alpha) u(-\vec{q}, \alpha)+\frac{1}{4 !} \sum_{\vec{q}_{1}, \vec{q}_{2}, \vec{q}_{3}, \vec{q}_{4}, \alpha} \lambda\left(\vec{q}_{1}, \vec{q}_{2}, \vec{q}_{3}, \vec{q}_{4}, \alpha\right) \\
& u\left(\vec{q}_{1}, \alpha\right) u\left(\vec{q}_{2}, \alpha\right) u\left(\vec{q}_{3}, \alpha\right) u\left(\vec{q}_{4}, \alpha\right) \delta\left(\vec{q}_{1}+\vec{q}_{2}+\vec{q}_{3}+\vec{q}_{4}\right)
\end{aligned}
$$

In the approximation of the mean field theory part of the normal mode coordinates may be replaced by their thermal average to obtain an effective interaction:

$$
\begin{aligned}
H_{W} & =\frac{1}{2} \sum_{\vec{q}, \alpha} \omega^{2}(\vec{q}, \alpha) u(\vec{q}, \alpha) u(-\vec{q}, \alpha) \\
& +\frac{1}{4} \sum_{\vec{q}, \vec{q}^{\prime}, \alpha} \lambda\left(\vec{q},-\vec{q}, \vec{q}^{\prime},-\vec{q}^{\prime}, \alpha\right)<u\left(\vec{q}^{\prime}, \alpha\right) u\left(-\vec{q}^{\prime}, \alpha\right)>u(\vec{q}, \alpha) u(-\vec{q}, \alpha)
\end{aligned}
$$

Here the mean fields $<u\left(\vec{q}_{1}, \alpha\right) u\left(\vec{q}_{2}, \alpha\right)>$ vanish due to crystal momentum conservation except for $\vec{q}_{1}=-\vec{q}_{2}$.

It should be noted that the terms $<u\left(\vec{q}^{\prime}, \alpha\right) u\left(-\vec{q}^{\prime}, \alpha\right)>u(\vec{q}, \alpha) u(-\vec{q}, \alpha)$ give the internal contributions to the required interaction Hamiltonian eq. (12) on the base space and thus to the desired gauge theories. Furthermore, these terms can be used to describe the phase transition induced by the $\eta$-mode: since eq. (42) is quadratic in the normal coordinates one may define effective frequencies $\Omega$ by

$$
\Omega^{2}(\vec{q}, \alpha)=\omega^{2}(\vec{q}, \alpha)+\frac{1}{2} \sum_{\vec{q}^{\prime}} \lambda\left(\vec{q},-\vec{q}, \vec{q}^{\prime},-\vec{q}^{\prime}, \alpha\right)<u\left(\vec{q}^{\prime}, \alpha\right) u\left(-\vec{q}^{\prime}, \alpha\right)>
$$


which fulfill the quasi-harmonic relation

$$
H_{W}=\frac{1}{2} \sum_{\vec{q}, \alpha} \Omega^{2}(\vec{q}, \alpha) u(\vec{q}, \alpha) u(-\vec{q}, \alpha)
$$

The redefined frequencies contain the leading order anharmonic effects and also the temperature dependence eq. (47) which will enter the description of the phase transition. The point is that the mean field $<u(\vec{q}, \alpha) u\left(\vec{q}^{\prime}, \alpha\right)>$ is the Fourier transform of the correlation function [34] and therefore can be given in terms of the phinon occupation number

$$
n(\Omega(\vec{q}, \alpha), T)=\frac{1}{\exp \left(\frac{\hbar \Omega(\vec{q}, \alpha)}{k_{B} T}\right)+1}
$$

as

$$
<u(\vec{q}, \alpha) u\left(\vec{q}^{\prime}, \alpha\right)>=\frac{\hbar}{\Omega(\vec{q}, \alpha)}\left[n(\Omega(\vec{q}, \alpha), T)+\frac{1}{2}\right]
$$

For $k_{B} T \geq \hbar \Omega(\vec{q}, \alpha)$ this is given to a very good approximation as

$$
<u\left(\vec{q}^{\prime}, \alpha\right) u\left(-\vec{q}^{\prime}, \alpha\right)>=\frac{k_{B} T}{\Omega^{2}\left(\vec{q}^{\prime}, \alpha\right)}
$$

To get a physical understanding let me assume that only one of the normal modes is responsible for the deformation and call the corresponding normal coordinate $\eta$. In fact this corresponds to the $A_{2 g}$ mode in the cases under consideration. In the high temperature phase it is just like any other normal mode, whereas near the critical point it freezes out and may be taken as the order parameter. Accordingly, eq. (42) can be put in the form

$$
H_{W}=V(\eta)+\frac{1}{2} \sum_{\vec{q}, \alpha}\left[\omega^{2}(\vec{q}, \alpha)+\frac{1}{2} g_{4}(\vec{q}) \eta^{2}\right] u(\vec{q}, \alpha) u(-\vec{q}, \alpha)
$$

where the quartic coupling of the soft mode to the other modes has been abbreviated as $g_{4}$.

The first term $V(\eta)$ in this equation contains the self interactions of the frozen mode. The corresponding couplings were called $-f_{2}=a\left(T_{c}-T\right)$ and $f_{4}$ in eq. (39) instead of $\omega^{2}$ and $\lambda$, and one sees that the frequency becomes imaginary at the transition point. The physical reason is that at low temperatures the structure of the high temperature phase is unstable with respect to the distortion of the soft mode and 
therefore the harmonic term of the mode must have a maximum for $\eta=0$. This can happen only, if the phinon frequency is imaginary. Above the transition point the high-symmetry phase is stable, because the anharmonic interactions increase the phinon frequency on increasing the temperature and the soft mode is an ordinary stable normal mode there.

To make contact between the microscopic theory and the Landau approach to phase transitions one can expand the standard expression for the phinon free energy eq. (8) in powers of $\eta$ :

$$
V_{F}=V_{F}(\eta=0)+\frac{1}{4} k_{B} T \sum_{\vec{q}} \frac{g_{4}(\vec{q})}{\Omega^{2}(\vec{q})} \eta^{2}+O\left(\eta^{4}\right)
$$

The 'Landau free energy' is defined as the contribution of the order parameter to the free energy. Taking the $\eta^{2}$ term in (49) as correction to the potential $V(\eta)$ eq. (39) one obtains the result for the Landau free energy:

$$
V_{L}(\eta)=-\frac{1}{2} f_{2} \eta^{2}+\frac{1}{4} k_{B} T \sum_{\vec{q}} \frac{g_{4}(\vec{q})}{\Omega^{2}(\vec{q})} T \eta^{2}+\frac{1}{4} f_{4} \eta^{4}+O\left(\eta^{6}\right)
$$

which can easily be put into the standard form

$$
V_{L}(\eta)=-\frac{1}{2} a\left(T-T_{c}\right) \eta^{2}+\frac{1}{4} f_{4} \eta^{4}+O\left(\eta^{6}\right)
$$

where

$$
a=\frac{1}{4} k_{B} \sum_{\vec{q}} \frac{g_{4}(\vec{q})}{\Omega^{2}(\vec{q})}
$$

and $T_{c}=-\frac{f_{2}}{a}$.

The model also can be used to calculate the behavior of the Landau free energy at low temperatures. Namely, the complete free energy is the sum of eqs. (39) and (8)

$$
V_{C}=-\frac{1}{2} f_{2} \eta^{2}+\frac{1}{4} f_{4} \eta^{4}+k_{B} T \sum_{\vec{q}, \alpha} \ln \left(2 \sinh \frac{\hbar \omega(\vec{q}, \alpha)}{2 k_{B} T}\right)
$$

which in the low-T limit takes the form:

$$
V_{C}=-\frac{1}{2} f_{2} \eta^{2}+\frac{1}{4} f_{4} \eta^{4}+\frac{1}{2} \sum_{\vec{q}, \alpha} \hbar \Omega(\vec{q}, \alpha)-k_{B} T \sum_{\vec{q}} \exp \left[-\frac{\hbar \Omega(\vec{q})}{2 k_{B} T}\right]
$$


This function takes its minimum at

$$
\eta^{2}=\frac{f_{2}}{f_{4}}-\frac{\hbar}{4 f_{4}} \sum_{\vec{q}} \frac{g_{4}(\vec{q})}{\Omega(\vec{q})}-\frac{\hbar}{4 f_{4}} \sum_{\vec{q}} \frac{g_{4}(\vec{q})}{\Omega(\vec{q})} \exp \left[-\frac{\hbar \bar{\omega}(\vec{q})}{2 k_{B} T}\right]
$$

where the first term is simply the minimum of the original double well potential, the second term corresponds to quantum corrections of the zero-point phinon motion at $T=0$ and the third term represents the leading temperature dependance at low temperatures. The quantum correction (second term) lowers the minimum, and increasing the temperature it is lowered even further up to $T=T_{c}$ where $\eta^{2}=0$.

I finish this section with the remark that the equations above hold only for the case that all entropy of the system is vibrational entropy. If there is a configurational order-disorder contribution to the phase transition[29, 28] this adds another term $\sim \eta^{2}$ to the Landau free energy (very similar in form to what the phinon contributes in the second term to eq. (50) ) and will thus modify the value of the transition temperature. Order-disorder phase transitions do not usually play an important role in vibrational problems but are more important in magnetic systems, with spin waves as quasi-particle excitations, and will thus influence the discussion presented in section 7 .

\section{Inner and Outer Parity Violation}

Parity violation of the weak interactions was discovered more than 50 years ago and still is one of the most puzzling effects known in physics. The fact that only lefthanded fermions (and righthanded antifermions) take part in the weak interactions should be considered a unique hint that inner symmetry and spacetime symmetry must be somehow related or mixed, in such a way that there is a parity violating contribution to the cross section of the form $\vec{p} \vec{s}$ where $\vec{p}$ is a fermion momentum and $\vec{s}$ its spin vector.

$\vec{p} \vec{s}$ is a pseudoscalar quantity built as a product of a vector and an axial vector, where the axial vector(=angular momentum) is itself a cross product of two vectors, so that $\overrightarrow{p s}$ has in fact the form of a scalar triple product like in eq. (29). More in general any pseudoscalar contribution to a cross section signals parity violation, because a pseudoscalar changes sign unter parity[24]. In low energy physics, parity 
violating effects usually arise from helical structures in molecules or crystals and can be described by pseudoscalars (similar to the above triple product) which measure the degree of parity violation[25, 24].

For the case of weak parity violation a microscopic interpretation is not available until today but there is a very successful effective description in terms of $\mathrm{V}$-A currents

$$
j_{\mu}^{V-A}=\bar{f}_{L} \gamma_{\mu} f_{L}=\bar{f} \gamma_{\mu} \frac{1}{2}\left(1-\gamma_{5}\right) f
$$

which are built into the Standard Model by the use of 'chiral fermions' $f_{L}=\frac{1}{2}(1-$ $\left.\gamma_{5}\right) f$ in the Lagrangian which exactly correspond to the V-A currents given above. Parity violation is thus described by explicit symmetry breaking terms, while in the left-right symmetric model mentioned earlier there is spontaneous breaking of parity via a rather complicated Higgs sector [1].

As a characteristic example of weak parity violation in the Standard Model consider the production and decay of a W-boson

$$
u\left(p_{u}\right)+\bar{d}\left(p_{d}\right) \rightarrow W^{+} \rightarrow \mu^{+}\left(p_{l}\right)+\nu_{\mu}\left(p_{n}\right)
$$

We shall work in the rest frame of the $\mathrm{W}$ and assume that the incoming up-quark beam defines the positive $\mathrm{x}$-direction. Assuming further relativistic fermions and a scattering angle $\beta$ one can parametrize the momenta as

$$
\begin{aligned}
p_{u}=\frac{m_{W}}{2}(1,1,0,0) & p_{d}=\frac{m_{W}}{2}(1,-1,0,0) \\
p_{n}=\frac{m_{W}}{2}(1, \cos \beta, \sin \beta, 0) & p_{l}=\frac{m_{W}}{2}(1,-\cos \beta,-\sin \beta, 0)
\end{aligned}
$$

where a righthanded coordinate system has been introduced with unit vectors $\vec{e}_{1}, \vec{e}_{2}$ and $\vec{e}_{3}$.

Due to the structure of the V-A currents which corresponds to the S-matrix element

$$
<W^{+}\left(p_{W}, \epsilon\right)|S| u\left(p_{u}\right) \bar{d}\left(p_{d}\right)>\sim \bar{v}\left(p_{d}\right) \epsilon_{\mu} \gamma^{\mu} \frac{1}{2}\left(1-\gamma_{5}\right) u\left(p_{u}\right)
$$

the $W^{+}$is produced with helicity -1 i.e. its angular momentum is antiparallel to the direction $\vec{p}_{u}$ of the up-quark and can therefore be described by the polarization vector for lefthanded circular polarization

$$
\vec{\epsilon}_{L}=\frac{1}{\sqrt{2}}(0,1,-i)=\frac{1}{\sqrt{2}}\left(\vec{e}_{2}-i \vec{e}_{3}\right)
$$


On the other hand, the matrix element for the decay of a W-boson with polarization $\vec{\epsilon}$ into two massless leptons

$$
<\mu^{+}\left(p_{l}\right) \nu\left(p_{n}\right)|S| W^{+}\left(p_{W}, \vec{\epsilon}\right)>\sim \bar{u}\left(p_{n}\right) \epsilon_{\mu} \gamma^{\mu} \frac{1}{2}\left(1-\gamma_{5}\right) v\left(p_{l}\right)
$$

leads to the rate

$$
\frac{d \Gamma}{d \Omega}\left(W^{+} \rightarrow \mu^{+}+\nu\right)=\frac{\alpha m_{W}}{32 \pi \sin ^{2}\left(\theta_{W}\right)}\left[1-\left(\hat{p}_{n} \vec{\epsilon}\right)\left(\hat{p}_{n} \vec{\epsilon}^{*}\right)-i\left(\vec{\epsilon} \times \vec{\epsilon}^{*}\right) \hat{p}_{n}\right]
$$

where $\hat{p}_{n}=\vec{p}_{n} /\left|\vec{p}_{n}\right|$ and the parity violating term is $-i\left(\vec{\epsilon} \times \vec{\epsilon}^{*}\right) \vec{p}_{n}$ and built from the triple product of 3 vectors. Note that in the case at hand the vector $i\left(\vec{\epsilon}_{L} \times \vec{\epsilon}_{L}^{*}\right)=-\vec{e}_{1}$ points into the negative $\mathrm{x}$-direction (the direction opposite to the direction of motion of the original up-quark). Note further, that the product $\vec{p} \vec{s}$ mentioned above will appear in the bremsstrahlung process $l^{ \pm} \rightarrow \nu_{l} W^{ \pm}$if the polarization vector $\vec{s}$ of the incoming lepton is known and that in fact the fermion polarisation vector $\vec{s}$ takes over the role of the cross product $i\left(\vec{\epsilon} \times \vec{\epsilon}^{*}\right)$ in eq. (62) .

Although these approaches and the resulting phenomenological predictions are able to describe accurately all the measured effects I do not think that they give an exhaustive and satisfactory explanation of the physics behind the phenomena. I will now consider the question whether the tetron model can lead us to a deeper understanding. What I want to do is to tie weak parity violation to the appearant chirality of the inner symmetry crystal. As repeatedly mentioned, its point group $A_{4} \times Z_{2}$ is 'chiral', i.e. it violates the parity of the internal space, and I will in fact try to construct in this section a connection between this inner chirality and the observed parity violation of the weak interaction.

The chirality of the crystal immediately implies that all phinon modes have to respect the helical structure of the system. Since after the symmetry breaking the $Z_{2}$-factor has nothing to do with inner parity any more, both the g- and the ustates in eq. (4) will reflect the inner helical structure. But how can this be related to external weak parity violation which is measured on chiral particles in physical space?

In order to answer this question one has to keep in mind, that internal and Minkowski space originate from a unified (6+1)-dimensional spacetime, which at the big bang has split into a physical and an inner symmetry part. Afterwards, there was the 
symmetry breaking, which led to a chiral geometry of the inner symmetry crystal, as described in section 5 .

To be definite I will use the 7-dimensional vector product to describe the effective interaction among the phinon excitations after the phase transition to $A_{4} \times Z_{2}$, and I will show that it can be used to describe the required correlation between the chirality of the internal lattice and the handedness of the weak interaction in physical space.

Before I proceed I want to review the known facts about the 7 -dimensional vector product: just as in 3 dimensions a vector product can be defined in 7 dimensions which assigns to any two vectors $\vec{v}$ and $\vec{w}$ in $R^{7}$ a vector $v \times w$ perpendicular to $\vec{v}$ and $\vec{w}$ and of magnitude $|\vec{v}||\vec{w}| \sin (\angle)$.

A cross product which fulfills these properties of bilinearity, orthogonality and magnitude exists in fact only in three and seven dimensions [30], a statement, which is related to Hurwitz's theorem, which says that normed division algebras can be defined only in 1, 2, 4 and 8 dimensions [36].

By definition, for the 7-dimensional vector product to work, one needs a sevendimensional euclidean space. However, I will identify one of these dimensions with time, i.e. work in $6+1$ dimensions with 3 dimensions being occupied by the internal lattice and the rest forming a 3+1 Minkowski spacetime. The notation will be Euclidean throughout with an implicit Wick rotation $\left(x_{0} \rightarrow i x_{0}\right)$ understood, whereby part of the vector product terms appearing later e.g. in eq. (64) become imaginary.

Unfortunately, the 7-dimensional cross product is less intuitive than the 3-dimensional one. For example, the choice of the perpendicular axis is not unique, which means that there are different possibilities to define the 7-dimensional cross product (which however turn out to be equivalent up to rotations of axes). One possibility to define is

$$
\vec{e}_{i} \times \vec{e}_{j}=\epsilon_{i j k} \vec{e}_{k}
$$

where $\epsilon_{i j k}$ is a completely antisymmetric tensor with a positive value +1 when ijk $=123,145,176,246,257,347,365[30]$. Equivalently, one may use the rules given in table 2. Assuming representations $\vec{v}=\sum_{i=1}^{7} v_{i} \vec{e}_{i}$ and $\vec{w}=\sum_{i=1}^{7} w_{i} \vec{e}_{i}$ one can then 


\begin{tabular}{|c|c|c|c|c|c|c|c|}
$\times$ & $\vec{e}_{1}$ & $\vec{e}_{2}$ & $\vec{e}_{3}$ & $\vec{e}_{4}$ & $\vec{e}_{5}$ & $\vec{e}_{6}$ & $\vec{e}_{7}$ \\
\hline$\vec{e}_{1}$ & 0 & $\vec{e}_{3}$ & $-\vec{e}_{2}$ & $\vec{e}_{5}$ & $-\vec{e}_{4}$ & $-\vec{e}_{7}$ & $\vec{e}_{6}$ \\
$\vec{e}_{2}$ & $-\vec{e}_{3}$ & 0 & $\vec{e}_{1}$ & $\vec{e}_{6}$ & $\vec{e}_{7}$ & $-\vec{e}_{4}$ & $-\vec{e}_{5}$ \\
$\vec{e}_{3}$ & $\vec{e}_{2}$ & $-\vec{e}_{1}$ & 0 & $\vec{e}_{7}$ & $-\vec{e}_{6}$ & $\vec{e}_{5}$ & $-\vec{e}_{4}$ \\
$\vec{e}_{4}$ & $-\vec{e}_{5}$ & $-\vec{e}_{6}$ & $-\vec{e}_{7}$ & 0 & $\vec{e}_{1}$ & $\vec{e}_{2}$ & $\vec{e}_{3}$ \\
$\vec{e}_{5}$ & $\vec{e}_{4}$ & $-\vec{e}_{7}$ & $\vec{e}_{6}$ & $-\vec{e}_{1}$ & 0 & $-\vec{e}_{3}$ & $\vec{e}_{2}$ \\
$\vec{e}_{6}$ & $\vec{e}_{7}$ & $\vec{e}_{4}$ & $-\vec{e}_{5}$ & $-\vec{e}_{2}$ & $\vec{e}_{3}$ & 0 & $-\vec{e}_{1}$ \\
$\vec{e}_{7}$ & $-\vec{e}_{6}$ & $\vec{e}_{5}$ & $\vec{e}_{4}$ & $-\vec{e}_{3}$ & $-\vec{e}_{2}$ & $\vec{e}_{1}$ & 0
\end{tabular}

Table 2: Multiplication rules for the 7-dimensional vector product. The unit vectors $\vec{e}_{i}$ can be identified with the octonion units via $\vec{e}_{1}=I, \vec{e}_{2}=J, \vec{e}_{3}=I J, \vec{e}_{4}=L$, $\vec{e}_{5}=I L, \vec{e}_{6}=J L, \vec{e}_{7}=(I J) L$ so as to fulfil the relation (66) $)$.

calculate e.g. the third component of the cross product of two vectors $\vec{v}$ and $\vec{w}$ as

$$
(\vec{v} \times \vec{w})_{3}=v_{1} w_{2}-v_{2} w_{1}+v_{4} w_{7}-v_{7} w_{4}-v_{5} w_{6}+v_{6} w_{5}
$$

A related ambiguity is that for any cross product $\vec{v} \times \vec{w}$ in $R^{7}$ there are other planes than that spanned by $\mathrm{v}$ and $\mathrm{w}$ giving the direction of the vector $\vec{v} \times \vec{w}$. This can be seen from eq. (63), where for any unit vector, which one chooses, there are three cross products of unit vectors, which takes its value (up to a sign). Each of these cross products corresponds to another plane mapped into the given direction.

To get a better understanding of rotations in higher dimensions, the notion of 'planes of rotation' is often introduced. A 'plane of rotation' is a 2-dimensional linear subspace of a given d-dimensional space which is mapped to itself by a given rotation. In 3 dimensions it is the uniquely determined plane perpendicular to the axis of rotation, whereas for general dimension $\mathrm{d}$ there can be up to [d/2] planes of rotation for a given rotation, i.e. the maximum number of such planes is $1,1,2,2,3,3$ etc for dimensions $2,3,4,5,6,7$ etc. If a rotation has several planes of rotation these are necessarily orthogonal to each other, with only the zero vector in common.

All these statements rely on an elementary result of linear algebra, namely that any orthogonal transformation in $R^{d}$ can be 'diagonalized' to a set of 2-dimensional 
rotations by angles $\theta_{i}, \mathrm{i}=1, \ldots,[\mathrm{d} / 2]$

$$
O_{2}=\left(\begin{array}{cc}
\cos \left(\theta_{i}\right) & \sin \left(\theta_{i}\right) \\
-\sin \left(\theta_{i}\right) & \cos \left(\theta_{i}\right)
\end{array}\right)
$$

plus at least one unity on the diagonal of the matrix, if $\mathrm{d}$ is odd. The planes of rotation are the 2-dimensional subspaces, on which these 2-dimensional rotations are defined.

Just as in 3 dimensions one may define a triple product as the scalar product of a vector with a cross product of 2 other vectors, cf. eq. (29). In 3 dimensions this pseudoscalar quantity gives the oriented volume of the parallelepiped spanned by the 3 vectors and is therefore invariant under the group $\mathrm{SO}(3)$ of proper rotations in 3 dimensions (but changes sign under parity). In 7 dimensions the situation is different [32]: The invariance group is not full $\mathrm{SO}(7)$ but the exceptional Lie group $G_{2} \subset \mathrm{SO}(7)$, which comprises the $S O(3) \times S O(3)$ symmetry of inner and physical space (and also the point group symmetries of the corresponding lattices).

There is an interesting and illuminating relation of the cross products in 3 and 7 dimensions with quaternions and octonions, respectively. They can in fact be related to the imaginary part of the product of two quaternions or octonions $\mathrm{V}$ and $\mathrm{W}[36]$. To see this one should identify the basis $\vec{e}_{i}, i=1, \ldots, 7$ of $R^{7}$ as used in eq. (63) with the octonion units $I, J, I J, L, I L, J L,(I J) L$ and then show that the cross product of two 7 -dimensional vectors is given by

$$
\vec{v} \times \vec{w}=\frac{1}{2} \operatorname{Im}(V W-W V)
$$

where on the right hand side octonion multiplication has been used on the imaginary octonions $\mathrm{V}$ and $\mathrm{W}, V=v_{1} I+v_{2} J+v_{3} I J+v_{4} L+v_{5} I L+v_{6} J L+v_{7}(I J) L$ (and similar for $\mathrm{W})$. Note that a change in sign in the definition of the associator $(I J) L=-I(J L)$ would reverse all PV effects.

At this point the reader may complain, that after all these elaborations there is still no real progress in interrelating internal and weak parity effects. After all, the rotations within two different planes of rotation, one internal and one in physical space, a priori are completely independent. However, we shall shortly see that the rules of the 7 -dimensional cross and triple products given in table 2 establish a connection between the rotary directions. 
To this end I come back to the concrete example of production and decay of a Wboson eq. (57). As before it is considered in the rest frame of the $\mathrm{W}$, and with relativistic fermions. In the present $3+3+1$ dimensional model let $P_{u}=\left(p_{u}, \vec{q}_{u}\right)$ be the 7 -momentum of the up-quark with $p_{u}$ given in eq. (58) and $\vec{q}_{u}$ the crystal momentum of the corresponding phinon in internal space, and similarly for the other particle momenta.

Concerning the parity issue the W-boson has two relevant planes of rotation. One is spanned by $\vec{e}_{2}$ and $\vec{e}_{3}$ and given by the left handed W-polarization eq. (60) which enters via the factor

$$
-i\left(\vec{\epsilon}_{L} \times \vec{\epsilon}_{L}^{*}\right)=\vec{e}_{2} \times \vec{e}_{3}=\vec{e}_{1}
$$

in eq. (62). The other one is the plane of rotation of the internal helix which any phinon mode has to respect and which in general will be left handed and spanned by two vectors $\vec{g}$ and $\vec{h}$. When the $W^{+}$is produced, they may be fixed without loss of generality to be given by $\vec{g}=\vec{e}_{5}$ and $\vec{h}=\vec{e}_{4}$ so that

$$
\vec{g} \times \vec{h}=-\vec{e}_{1}
$$

according to table 2 .

The situation may be compared to that in optical activity of chiral molecules or crystals, where the polarized photon induces a current in the chiral geometry, which in turn influences the photon in such a way that its polarization is rotated. Geometrically this is due to the interplay of two chiral structures: one from the polarized photon, and the other one from the chiral object.

In the present case the two chiral objects a priori are independent, because they live in two different subspaces of the $6+1$ dimensional world. The plane spanned by $\vec{g}$ and $\vec{h}$ as well as the crystal-momenta of the phinon modes $\vec{q}_{u}=-\vec{q}_{d}$ are all orthogonal to physical space. However, the rules of the 7-dimensional vector product in table 2 are such that they give $\vec{g} \times \vec{h}=\vec{e}_{5} \times \vec{e}_{4}=-\vec{e}_{1}$, i.e. they put the internal helical structure in parallel with the external angular momentum $i\left(\vec{\epsilon}_{L} \times \vec{\epsilon}_{L}^{*}\right)=-\vec{e}_{1}$ and in general forbid $\vec{g} \times \vec{h}$ to point into one of the inner directions. It is only due to this peculiar structure of the 7-dimensional vector product that the two chiralities which in principle live in two different subspaces can meet in the form of an interaction which ties them together. In fact, when the $W^{+}$decays to $\mu^{+}\left(P_{l}\right)+\nu_{\mu}\left(P_{n}\right)$, the 
plane of the internal helix will become directed towards the neutrino momentum

$\vec{g} \times \vec{h}=-\hat{p}_{n}$, and the parity violating term in the Standard Model prediction eq. (62) can be reconstructed from a matrix element, which is the product of an internal contribution $<q_{W}\left|S_{i n}\right| q_{l} q_{n}>$ and a spatial contribution $<p_{W}\left|S_{s p}\right| p_{l} p_{n}>$, as

$$
\left[-i\left(\vec{\epsilon}_{L} \times \vec{\epsilon}_{L}^{*}\right) \hat{p}_{n}\right]\left[(\vec{g} \times \vec{h}) \hat{p}_{n}\right]=-i\left(\vec{\epsilon}_{L} \times \vec{\epsilon}_{L}^{*}\right)(\vec{g} \times \vec{h})=\vec{e}_{1} \hat{p}_{n}
$$

The interaction given by the 7-dimensional vector product is antisymmetric under odd permutations in both its internal and physical part. This is precisely what is needed to describe the parity violation, simply because one will get negative energy contributions to the partition function, if an odd transformation is applied to the points of a tetrahedron.

The 7-dimensional triple product will thus give a good description of the parity violating effect. However, it should be kept in mind, that this description is only an effective one, because it is originally induced by the chiral geometric structure of the internal lattice. The geometrical structure comes first, so that when two phinons collide they have to respect the chirality of the $A_{4}$ symmetry in such a way that only spatially lefthanded states can enter the game. What the 7 -dimensional triple product does, is to to connect the parity effects in internal and physical space in such a way that the chiral nature of the inner symmetry lattice induces parity violation in physical space.

\section{The Mignon Alternative}

There is another interpretation of the tetrahedral ordering of the spectrum eq. (4), namely by using the rotational instead of the translational transformation properties in the last column of table 1. In that case one is led to consider spin models, and the vector displacements $\vec{u}$ of the atomic vibrations are replaced by axial vectors $\vec{S}$ which fulfil angular momentum commutation relations.

Spin models have been considered in statistical and solid state physics for a long time [12, 13, 45], and they have been used to describe magnetic phase transitions and excitations as well as many other phenomena. In contrast to ordinary spin models, which were first used by Heisenberg to describe ferromagnetic effects in solids, the 
spin models in this section are defined on inner symmetry space, because I want to use them as an alternative to the phinon picture developed in the preceeding sections. Phinons are then to be replaced by 'mignons', quasi-particle excitations which differ from magnons by living on the internal $A_{4} \times Z_{2}$ crystal structure, i.e. they are defined with the help of an internal 3-dimensional 'spin vector' $\vec{S}(m)$ given on lattice points $\mathrm{m}$ and interacting via a Heisenberg like internal Hamiltonian

$$
H_{H}=-\sum_{m, m^{\prime}} J\left(m, m^{\prime}\right) \vec{S}(m) \vec{S}\left(m^{\prime}\right)
$$

where $J\left(m, m^{\prime}\right)$ are the coupling strengths and the sum runs over all neigbouring lattice sites. Depending on the sign of J one has ferromagnetic- or antiferromagnetc type of interactions. In contrast to phinons, which arise from vibrational displacement vectors $\vec{u}$, the spin vectors are (internal) axial vectors and have (internal) angular momentum properties, as will be shown below.

The physics behind such a model relies on exchange integrals of fermion wave functions $\psi_{ \pm \frac{1}{2}}$ defined on the inner crystal of the form

$$
I_{E}=\frac{1}{2} \int d^{3} r_{1} d^{3} r_{2} \sum_{\alpha, \beta=1}^{2} \psi_{\alpha}^{\dagger}\left(\vec{r}_{1}\right) \psi_{\beta}^{\dagger}\left(\vec{r}_{2}\right) V\left(\left|\vec{r}_{1}-\vec{r}_{2}\right|\right) \psi_{\beta}\left(\vec{r}_{1}\right) \psi_{\alpha}\left(\vec{r}_{2}\right)
$$

where the sums run over 2 internal 'spin' directions $\alpha, \beta= \pm \frac{1}{2}, \mathrm{~V}$ is an internal potential and $\vec{r}_{i}$ are vectors in internal space. These exchange integrals can be shown to lead directly to the Heisenberg Hamiltonian eq. (70) while other possible contributions which may look interesting for chiral crystal structures like the the Dzyaloshinsky-Moriya interactions [41, 42] $\vec{D}\left(m, m^{\prime}\right)\left[\vec{S}(m) \times \vec{S}\left(m^{\prime}\right)\right]$ are not generated in leading order. They can arise e.g. by spin-orbit corrections and are usually suppressed.

The following relation constitutes the link between the internal spin vector $\vec{S}$ in eq. (70) and the internal spinor $\psi$ in eq. (71):

$$
\vec{S}=\frac{1}{2} \psi^{\dagger} \vec{\tau} \psi
$$

where $\vec{\tau}$ is the triplet of internal Pauli matrices.

The possibility of a $6+1$ dimensional discrete spacetime which soon after the big bang got split to $3+3+1$ dimensions has been repeatedly discussed in the previous 
sections, and I will in fact assume in the following that the original dynamical field is a (6+1)-dimensional spinor, whose 'mignon' excitations on the inner symmetry space can be identified with the quark, lepton and gauge boson fields appearing in the Standard Model.

Such a spinor can be considered a $\mathrm{SO}(7)$ spinor by a Wick rotation 5 and to two $\mathrm{SO}(6)$ spinors (one for a $\mathrm{SO}(6)$ fermion and the other for an antifermion) in the non-relativistic limit. In general, higher dimensional spinors can be constructed from building blocks of spinors in lower dimensions, as described for example in ref. [39]. In the case at hand, they have a close relationship to the octonion algebra [35], just as 3-dimensional spinors have to quaternions. While the Pauli matrices can be identified more or less directly with the quaternion units, the situation in 7 or $6+1$ dimensions is somewhat more subtle because the octonion algebra is not associative, i.e. the octonion units I, J, IJ, L, IL, JL and (IJ)L cannot be exactly represented by 7-dimensional matrices. A detailed and explicit description of the relationship can be found, for example, in the book by Dixon [35].

While the covering group of $\mathrm{SO}(6)$ is isomorphic to $\mathrm{SU}(4)$ and has 2 fundamental complex representations $\underline{4}$ and $\underline{4}^{*}$ which are conjugate to each other, the covering group of $\mathrm{SO}(7)$ is $\operatorname{Spin}(7)$ with one spinor representation of dimension $\underline{\mathbf{8}}$, which is again related to the non-associative division algebra of octonions [35, 36, 37, 38]. Breaking $\operatorname{Spin}(7) \rightarrow \mathrm{SU}(4)$ there is a decomposition $\underline{8} \rightarrow \underline{4}+\underline{4}^{*}$ which reveals the particle antiparticle content of the original $\mathrm{SO}(6,1)$ spinor [39].

For simplicity let me concentrate on the nonrelativistic $\mathrm{SO}(6)$ fermion. The covering group of $\mathrm{SO}(6)$ is $\mathrm{SU}(4), \widetilde{S O(6)}=S U(4)$. Dividing it into a spatial and an inner symmetry part $S U(2)^{s p} \times S U(2)^{i n}$ reduces its spinor representation $\underline{\mathbf{4}} \rightarrow\left(\underline{\mathbf{2}^{s p}}, \underline{\mathbf{2}^{i n}}\right)$ further to a field $\phi_{a, \alpha}$ which is a doublet both under $S O(3)^{s p}$ and under $S O(3)^{i n}$, i.e. the index a acts as a Pauli spinor index in physical space whereas $\alpha$ is a 'spinor' index in the inner symmetry space. Going to an inner lattice with $A_{4}$ symmetry, i.e. a space with symmetry group $S O(3)^{s p} \times A_{4}^{i n}$, one obtains states which transform as $\left(\underline{\mathbf{2}^{s p}}, G_{1}^{i n}\right)$ where $G_{1}$ is the 2-dimensional spinor representation of $A_{4}$ obtained by restricting the $\underline{2^{i n}}$ representation of $S U(2)^{i n}=\widetilde{S O(3)^{i n}}$ to $A_{4}^{i n}$. If physical space has

\footnotetext{
${ }^{5}$ More generally, in $S O\left(d_{1}, d_{2}\right)$ the spinor dimensions viewed over complex space coincide with the case of the $\left(d_{1}+d_{2}\right)$-dimensional Euclidean space.
} 


\begin{tabular}{|c|c|c|}
\hline the fundamental fermion & $\operatorname{Spin}(7) \leftrightarrow \widetilde{S O(6,1)}$ & $\underline{\mathbf{8}}=F$ \\
\hline nonrelativistic limit & $\widetilde{S O(6,1) \rightarrow S U(4)}$ & $\underline{\mathbf{8}} \rightarrow \underline{\mathbf{4}}+\underline{\mathbf{4}}^{*}$ \\
\hline inner and physical space split & $S U(4) \rightarrow S U(2) \times S U(2)$ & $\underline{\mathbf{4}} \rightarrow\left(\mathbf{2}_{\boldsymbol{i n}}, \mathbf{2}_{\boldsymbol{s p}}\right)=\phi_{a, \alpha}$ \\
\hline restriction to a lattice & $S U(2) \times S U(2) \rightarrow \widetilde{S_{4}^{s p}} \times \widetilde{S_{4}^{\text {in }}}$ & $\underline{\mathbf{4}} \rightarrow\left(G_{1}^{s p}, G_{1}^{\text {in }}\right)$ \\
\hline
\end{tabular}

Table 3: Group theoretic view on the fundamental dynamical field $\mathrm{F}$ in the tetron model. The tilde denotes covering groups.

a lattice structure, too, one ends up with $\left(G_{1}^{s p}, G_{1}^{i n}\right)$. The overall group theoretic situation is summarized in table 3 .

We now make the further assumption that the compactification process completely separates the interactions in physical and internal space. Then the eigenfunctions $\phi_{a, \alpha}$ will factorize as $\phi_{a, \alpha}=f_{a} \psi_{\alpha}$ where $\psi_{\alpha}$ constitutes the internal spin vector $\vec{S}$ according to eq. (72), which will follow the dynamics of the inner Heisenberg model eq. (70), while the physical spinor part $f_{a}$ (or its relativistic extension) fulfills the Dirac equation. The corresponding Bloch waves are a product of an internal spin wave (='mignon') and a free Dirac wave

$$
\Psi_{i a}=f_{a}(\vec{x}) S_{i}(\vec{r}) e^{i(\vec{p} \vec{x}-\nu t)} e^{i(\vec{q} \vec{r}-\omega t)}
$$

i.e. at a given point the free Dirac wave must be multiplied by the mignon mode. If given on different spots $m$ and $m^{\prime}$ the inner spin vectors commute; however given on the same spot $m$ they fulfil angular momentum commutation relations

$$
\left[S_{i}(m), S_{j}(m)\right]=\epsilon_{i j k} S_{k}(m)
$$


where $i, j, k \in 1,2,3$ denote the 3 directions in the internal crystal.

The equations of motion for the operators $\vec{S}(m)$ can be obtained most easily in the Heisenberg picture:

$$
\frac{d \vec{S}(m)}{d t}=\frac{i}{\hbar}\left[H_{H}, \vec{S}(m)\right]
$$

Inserting $H_{H}$ from eq. (70) leads to

$$
\frac{d S_{i}(m)}{d t}=-\epsilon_{i j k} \sum_{m^{\prime}} J\left(m, m^{\prime}\right) S_{j}(m) S_{k}\left(m^{\prime}\right)
$$

These equations decouple, if one defines $S_{ \pm}(m)=S_{1}(m) \pm i S_{2}(m)$, and one can show that there are plain wave solutions of the form

$$
S_{ \pm}(m)=S_{ \pm}(\vec{q}) e^{i(\vec{q} r(m)-\omega t)}
$$

which are called internal spin waves or mignons. More precisely, the $\mathrm{x}$ - and $\mathrm{y}$ components turn out to be out of phase by an angle $\pi / 2$, so that $S_{1}(t)$ and $S_{2}(t)$ can be said to precess on a cone.

Just as for phinons the next step is to consider nonlinear effects which lead to interactions among mignons, to scattering processes and possibly to bound states. For the following discussion it is convenient to switch to the language of the second quantization. We could have done so for phinons as well, however at will, whereas here it is really advisable. The point is, that as bilinears of internal fermions the spin operators $S_{i}$ and the associated quasi-particles should be internal bosons. However, as they stand they do not fulfil the canonical commutation relations for bosons, and this can be remedied most easily in the framework of the second quantization. Namely, in order to recover the ordinary boson commutation relations, they may be rewritten with the help of a Holstein-Primakoff transformation[49] as

$$
\begin{aligned}
S_{+}(m) & =\sqrt{2 S-a^{\dagger}(m) a(m)} a(m) \\
S_{-}(m) & =a^{\dagger}(m) \sqrt{2 S-a^{\dagger}(m) a(m)} \\
S_{z}(m) & =S-a^{\dagger}(m) a(m)
\end{aligned}
$$

One can prove that the commutation relations eqs. (74) are satisfied for this representations iff the creation and annihilation operators $a^{\dagger}(m)$ and $a(m)$ fulfil the canonical commutation relations for harmonic oscillators. 
The spin $\mathrm{S}$ in eq. (80) is defined by $\vec{S}(m) \vec{S}(m)=S(S+1) \mathbf{1}$ and the products $a^{\dagger}(m) a(m)$ are number operators with eigenvalues $0,1,2, \ldots$. Since $S_{z}(m)$ is supposed to take eigenvalues $-S, \ldots S-1, S$ one has to impose the constraint $a^{\dagger}(m) a(m) \leq 2 S$. This inconvenience is compensated for by the fact that the vacuum of the HolsteinPrimakoff bosons fulfills 49 ]

$$
a^{\dagger}(m) a(m)\left|0(m)>=0 \quad \Longleftrightarrow \quad S_{z}(m)\right| 0(m)>=S \mid 0(m)>
$$

and can thus be identified with the 'ferromagnetic' ground state.

For practical calculations the roots appearing in eq. (80) should be expanded in powers $a^{\dagger}(m) a(m) / 2 S$. Using momentum space creation and annihilation operators

$$
\begin{aligned}
a(m) & =\sum_{\vec{q}} \exp [i \vec{q} \vec{r}(m)] b(\vec{q}) \\
a^{\dagger}(m) & =\sum_{\vec{q}} \exp [-i \overrightarrow{q r}(m)] b^{\dagger}(\vec{q})
\end{aligned}
$$

the Hamiltonian of the system can be expressed as

$$
H_{H}=E_{0}+\sum_{\vec{q}} \hbar \omega(\vec{q}) b^{\dagger}(\vec{q}) b(\vec{q})+\sum_{\vec{q}, \vec{q}^{\prime}} V\left(\vec{q}, \vec{q}^{\prime}\right) b^{\dagger}\left(\vec{q}^{\prime}\right) b^{\dagger}\left(-\vec{q}^{\prime}\right) b(\vec{q}) b(-\vec{q})
$$

where $E_{0}$ is the ground state energy and it has been assumed that stable mignon pairs exist only if the two mignons have opposite wave vectors.

Turning now to the special case of the $A_{4} \times Z_{2}$ lattice under discussion in this paper, one may approximately introduce two coupling constants, called J and J', one which couples atoms within the lattice tetrahedrons and one which couples two tetrahedrons (or equivalently a $Z_{2}$-pair). As in section 3 one may examine the limiting cases of large $\mathrm{J}$ and large J', respectively, and perform a calculation within the mean field approximation. For that one should use appropriate local states, dublet states $\left.\frac{1}{2}(|\uparrow> \pm| \downarrow\rangle\right)$ for the $Z_{2}$ excitations and 16 states built from $\mid \uparrow \uparrow \uparrow \uparrow>$, $\mid \uparrow \uparrow \uparrow \downarrow>$ etc for a tetrahedron.

Within this framework the following bilinears can be replaced by their thermal averages:

$$
\begin{aligned}
b(\vec{q}) b(-\vec{q}) & =\langle b(\vec{q}) b(-\vec{q})> \\
b^{\dagger}(\vec{q}) b^{\dagger}(-\vec{q}) & =\left\langle b^{\dagger}(\vec{q}) b^{\dagger}(-\vec{q})>\right.
\end{aligned}
$$


so that the interaction part of the Hamiltonian can be approximated by

$$
\begin{aligned}
H_{H}= & E_{0}+\frac{1}{2} \sum_{\vec{q}} \hbar \omega(\vec{q})\left[b^{\dagger}(\vec{q}) b(\vec{q})+b^{\dagger}(-\vec{q}) b(-\vec{q})\right] \\
& +\sum_{\vec{q}, \vec{q}^{\prime}} V\left(\vec{q}, \vec{q}^{\prime}\right)\left[b^{\dagger}\left(\vec{q}^{\prime}\right) b^{\dagger}\left(-\vec{q}^{\prime}\right)<b^{\dagger}(\vec{q}) b^{\dagger}(-\vec{q})>\right. \\
& \left.+b(\vec{q}) b(-\vec{q})<b^{\dagger}\left(\vec{q}^{\prime}\right) b^{\dagger}\left(-\vec{q}^{\prime}\right)>-<b(\vec{q}) b(-\vec{q})><b^{\dagger}\left(\vec{q}^{\prime}\right) b^{\dagger}\left(-\vec{q}^{\prime}\right)>\right]
\end{aligned}
$$

This result may be used as a starting point to analyze phase transitions and mignonmignon scattering processes, very similar to eq. (42) for phinons. For example, using shifted frequencies

$$
\Omega(\vec{q})=\omega(\vec{q})\left[1+\frac{E_{0}}{8} \sum_{\vec{q}^{\prime}} \hbar \omega\left(\vec{q}^{\prime}\right)<b(\vec{q}) b(-\vec{q})>\right]
$$

one may put $H_{H}$ into the form 43

$$
H_{H}=E_{0}+\frac{1}{2} \sum_{\vec{q}} \hbar \Omega(\vec{q})\left[b^{\dagger}(\vec{q}) b(\vec{q})+b^{\dagger}(-\vec{q}) b(-\vec{q})\right]
$$

\section{Conclusions}

In the present paper the tetron model of elementary particles has been further developed, and arguments have been given how some old puzzles of high energy physics can be explained by giving a discrete structure to a 3-dimensional inner symmetry space, which has the form of a finite internal crystal lattice with 8 points in its unit cell.

The fermions and gauge bosons of the Standard Model have been interpreted as quasi-particle excitations, which arise on this lattice from vibrations or quantum exchange of more fundamental entities. Since the phenomenological indications are not conclusive, I have analyzed two kinds of models, one describing vibrational excitations on the internal space (called 'phinons'), the other one with 'mignons'. Bound states from these excitations have been examined as well as symmetry breaking scenarios, which may have led to phase transitions in the early universe.

It is an old dream of theoretical physicists that inner symmetries may be obtained by extending ordinary space to higher dimensions (see for example ref. [31]). The 
present paper shows how the connection between inner and outer symmetries has to look like in order to handle the observed parity violation of the weak interactions. It is argued that at the big bang the universe started with a unified discrete $6+1$ dimensional spacetime. Afterwards it underwent at least 2 phase transitions: in the first it split into a physical and a compact internal part, where the latter cannot be directly perceived, but is only observable by effect of charges and interactions. At a later stage, when physical and internal space cooled down further towards the weak interaction scale, there was a phase transition within the internal lattice, which led to a chiral structure with point group $A_{4} \times Z_{2}$.

As explained in footnote 1, I have not been definite about using a discrete base spacetime or not. However, if one is willing to make this additional assumption, one encounters advantages as well as drawbacks. The advantages are that ultraviolet divergences are regularized per se and no renormalization is needed when it comes to calculations involving short distances. Furthermore, no-go theorems like the Weinberg-Witten theorem [48] which in the continuum forbid the unification of spatial and inner symmetries do not apply. Finally, of course, the lattice ansatz naturally explains the selection rule mentioned in ref. [2] that all physical states must be permutation states: just because the lattice excitations must transform under representations of its point group $A_{4} \times Z_{2}$.

A credit point not mentioned at all in this paper is that a lattice ansatz, which not only discretizes internal but also physical space, naturally explains why there are no fundamental particles with spin larger than 2 in nature: this has to do with the fact that the discrete symmetry groups under discussion possess only irreducible representations with small dimensions. For example, assuming a cubic spatial lattice with $S_{4}^{s p}$ as a point group, there are (ray)-representations of dimension $1\left(A_{1}, A_{2}\right)$, $2\left(G_{1}, G_{2}, E\right), 3\left(T_{1}, T_{2}\right)$, and $4(H)$. $G_{1}$ has been discussed as the relevant spatial spin-1/2 representation for fermions in the last section, but one may justly ask the question, why the other modes, like e.g. the spin-3/2 representation $G_{2}+H$ are not observed. This question has been addressed in a separate publication[16], where those modes were suggested as dark matter candidates.

Now for the drawbacks: first of all, on a spatial lattice Lorentz symmetry is broken, which is of course in contradiction to everyday experience. However, for a classical 
observer Lorentz symmetry can be restored by assuming the lattice spacing to be either unobservably small or that it may be a fluctuating quantum lattice, where the lattice points move around randomly and follow some quantum stochastic process [44]. There is some relation of this idea to other models which involve a fundamental length scale, like quantum foam models [40, 46, 47], which however assume gravity to play the central role in the dynamics, while in the present model gravitational interactions and cosmological phenomena appear only as byproducts of the tetron lattice interactions, as shown in ref. [16].

Secondly, it is usually difficult to define fermions on a lattice without getting problems with (micro)causality, because in contrast to bosons fermions 'know' about other fermions on neighbouring lattice sites and this induces nonlocal correlations and possible synchronisations beyond the event horizon. Fortunately, this is not the case in the approach presented here, because the fundamental fermion field introduced in the last section is defined within the whole crystal (both for internal and physical space) not just on the lattice points.

I have given arguments how the gauge structure and also the parity violation of the weak interaction have arisen from properties and interactions within the internal crystal. At the present stage, the true nature of the underlying dynamics that controls the vibrational or 'magnetic' excitations remains unclear. For example, it is possible that it turns out to be in some sense supersymmetric. However, one should consider this option far from being compelling. In particular, the appearance of discrete lattice structures above Planck distances gives no indication that the fundamental Lagrangian will have anything to do with the nowadays popular superstring or M-theories.

\section{References}

[1] R. N. Mohapatra and J. C. Pati, Phys. Rev. D11 (1975) 566, 2558.

[2] B. Lampe, Found. Phys. 39 (2009) 215.

[3] B. Lampe, J. Phys. G 34 (2007) 1 and arXiv:hep-ph/0610270 (2006); see also arXiv:hep-ph/9810417 (1998). 
[4] B. Lampe, Mod. Phys. Lett. A23 (2008) 2835 and arXiv:0805.3762v1 (2008).

[5] D.L. Rousseau, R.P. Bauman, S.P.S. Porto, J. Raman Spectr., 10 (1981) 253.

[6] E. Kroumova, M. I. Aroyo, J. M. Perez Mato, A. Kirov, C. Capillas, S. Ivantchev, H. Wondratschek, Phase Transitions 76 (2003) 155.

[7] R.C. Johnson, Phys. Lett. 114B (1982) 147.

[8] A. A. Belavin, A. M. Polyakov, A. B. Zamolodchikov, Nucl. Phys. B 241 (1984) 333.

[9] J.S. Griffith, Irreducible Tensor Methods for Molecular Symmetry Groups, Dover Publications, 2006.

[10] H.U. Besche, B. Eick, and E.A. O'Brien, Internat. J. Alg. and Comp. 12 (2002) 623.

[11] H.U. Besche, B. Eick, Elec. Res. Announcements Amer. Math. Soc. 7 (2001) 1

[12] C. Herring, C. Kittel, Phys. Rev. 81 (1951) 869

[13] F.J. Dyson, Phys. Rev. 102 (1956) 1217

[14] D. Alfe, Comp. Phys. Comm. 180 (2009) 2622.

[15] H.T. Stokes, L.L. Boyer (2002) www.physics.byu.edu/ stokesh/isotropy.htm

[16] B. Lampe, Cent. Eur. J. Phys. 2, 193 (2010).

[17] A. Hasenfratz, P. Hasenfratz, K. Jansen, J. Kuti, Y. Shen, Nucl. Phys. B365 (1991) 79.

[18] S. Dimopoulos, L. Susskind, Nucl. Phys. B155 (1979) 237.

[19] S. Uehara, T. Yanagida, Phys. Lett. B165 (1985) 94.

[20] D. Amati, R. Barbieri, A.C. Davis, G. Veneziano, Phys. Lett. B102 (1981) 408.

[21] J. Smit, Nucl. Phys. B175 (1980) 307.

[22] P. Maraner, J.K. Pachos, arXiv:0807.0826v2 (2009). 
[23] F. Berruto, G. Grignani, G.W. Semenoff, P. Sodano, Phys. Rev. D57 (1998) 5070.

[24] G.T. Pickett, M. Gross, H. Okuyama, Phys. Rev. Lett. 85 (2000) 200.

[25] A.B. Harris, R.D. Kamien, T.C. Lubensky, arXiv:cond-mat/9901174v2 (1999).

[26] C.E. Cordeiro, E. V. de Mello und M. A. Continentino, Z. Phys. B85 (1991) 307.

[27] W. Cochran, Phys. Rev. Lett, 3 (1959) 412.

[28] E.K.H. Salje, B. Wruck, H. Thomas, Zeitschr. f. Phys. B82 (1991) 399.

[29] P. Sollich, V. Heine, M.T. Dove, J. Phys., 6 (1994) 3171.

[30] W.S. Massey, Am. Math. Monthly 10 (1990) 697.

[31] V. H. S. Kumar and P. K. Suresh, arXiv:gr-qc/0605016.

[32] S. L. Cacciatori and A. Scotti, arXiv:hep-th/0503106.

[33] M. T. Dove, American Mineralogist, 82 (1997) 213.

[34] M. T. Dove, T. Cool, D.C. Palmer, A. Putnis, E.K.H. Salje and B. Winkler, American Mineralogist, 78 (1993) 486.

[35] G.M. Dixon, Division Algebras, Kluwer Books, 2009.

[36] J. Conway and D. Smith, On Octonions and Quaternions, Peters Publishing, Natick, MA (2003).

[37] I. L. Kantor and A. S. Solodovnikov, Hypercomplex Numbers - an Elementary Introduction to Algebras, Springer Verlag, Berlin, 1989.

[38] R.D. Schafer, An Introduction to Nonassociative Algebras, Academic Press, New York and London, 1966.

[39] G. G. Ross, Grand Unified Theories, Oxford University Press, 1984.

[40] K. Ford and J. A. Wheeler, Geons, Black Holes and Quantum Foam 1995 [ISBN 0-393-04642-7]. 
[41] I. Dzyaloshinsky, J. Phys. Chem. Solids 4 (1958) 241.

[42] T. Moriya, Phys. Rev. 120 (1960) 91.

[43] J.J. Zhang, J.H. Yuan, Q.J. Zeng, J.P. Zhang, Commun. Theor. Phys. 56 (2011) 173

[44] R. J. Adler and D. I. Santiago, Mod. Phys. Lett. A 14 (1999).

[45] X.-G. Wen, cond-mat/0107071 (2001)

[46] J. Ambjorn, J. Jurkiewicz and R. Loll, arXiv:hep-th/0002050.

[47] H. Yamamoto, Phys. Rev. D30 (1984) 1727.

[48] S. Weinberg and E. Witten, Phys. Lett. B96 (1980) 59.

[49] T. Holstein and H. Primakoff, 58 (1940) 1098. 